\title{
Chemical design of self-propelled Janus droplets
}

Caleb H. Meredith ${ }^{1}$, Alexander Castonguay ${ }^{2}$, Yu-Jen Chiu', Allan M. Brooks ${ }^{3}$, Pepijn Moerman ${ }^{4}$, Peter Torab $^{5}$, Pak Kin Wong ${ }^{5,6,7}$, Ayusman Sen ${ }^{2,3}$, Darrell Velegol ${ }^{3}$, Lauren D. Zarzar ${ }^{1,2,8^{*}}$

1. Department of Materials Science and Engineering, The Pennsylvania State University, University Park, PA 16802, USA

2. Department of Chemistry, The Pennsylvania State University, University Park, PA 16802, USA

3. Department of Chemical Engineering, The Pennsylvania State University, University Park, PA 16802, USA

4. Department of Chemical and Biomolecular Engineering, Johns Hopkins University, Baltimore, MD 21218, USA

5. Department of Mechanical Engineering, The Pennsylvania State University, University Park, PA 16802, USA

6. Department of Biomedical Engineering, The Pennsylvania State University, University Park, PA 16802, USA

7. Department of Surgery, College of Medicine, The Pennsylvania State University, Hershey, PA 17033, USA

8. Materials Research Institute, The Pennsylvania State University, University Park, PA 16802, USA

*Lauren D. Zarzar

Email: Idz4@psu.edu

Author Contributions: C.H.M. and L.D.Z. developed the concept for research. C.H.M, A.C., Y-J.C., and L.D.Z. designed and conducted experiments related to droplet fabrication, speed and shape analysis, and chemical properties. A.C. analyzed the behavior of droplets under thermal gradients. A.M.B., A.S., D.V. conducted video analysis of droplets to extract chemical trail interactions. P.M. conducted particle tracking for flow analysis. P.T., P.K.W. designed and fabricated electrode heaters and analyzed thermal profiles.

Competing Interest Statement: Authors declare no competing interests.

Classification: Physical sciences - chemistry

Keywords: Janus droplets, self-propulsion, active matter, collective behavior, solubilization

This PDF file includes:

Main text

Figure 1-6

\section{Abstract}

The study of active colloidal microswimmers with tunable phoretic and self-organizational behaviors is important for understanding out-of-equilibrium systems and the design of functional, adaptive matter. Solubilizing, self-propelling droplets have emerged as a rich chemical platform for exploration of active behaviors, but isotropic droplets rely on spontaneous symmetry breaking to sustain motion. The introduction of permanent asymmetry, e.g. in the form of a biphasic Janus droplet, has not been explored previously as a comprehensive design strategy for active droplets, despite the widespread use of Janus structures in motile solid particles. Here, we uncover the chemomechanical framework underlying the self-propulsion of biphasic Janus oil droplets solubilizing in aqueous surfactant. We elucidate how droplet propulsion is influenced by the degree of oil mixing, droplet shape, and oil solubilization rates for a range of oil combinations. A key finding is that for droplets containing both a mobile (solubilizing) and non-mobile oil, the degree of partitioning of the mobile oil across the Janus droplets' oil-oil interface plays a pivotal role in 
determining the droplet speed and swimming direction. In addition, spatiotemporal control over droplet swimming speed and orientation is demonstrated through the application of local thermal gradients applied via joule heating and laser illumination. We also explore the interactions between collections of Janus droplets including the spontaneous formation of multi-droplet clusters that spin predictably based on symmetry. Our findings provide insights as to how the chemistry and structure of multiphase fluids can be harnessed to design microswimmers with programmable active and collective behaviors.

\section{Significance Statement}

Active materials capable of autonomous movement are of interest for understanding and harnessing the emergent properties of out-of-equilibrium systems. Multiphase fluids, such as emulsions, are an exciting platform for producing increasingly complex active colloids given their liquid-liquid interfaces which enable dynamic control over chemical transport and partitioning between fluid compartments. Here, we investigate the active behavior and underlying chemomechanical mechanisms imparting motility to microscale, self-propelled, solubilizing Janus oil droplets. A key discovery is that the two compartments of the biphasic Janus droplets function cooperatively via oil partitioning to govern droplet speed, direction, and propulsion efficiency.

\section{Main Text}

\section{Introduction}

Active, colloidal microswimmers of diverse compositions and propulsion mechanisms are of interest as minimal models to probe out-of-equilibrium behavior and collective organization. For a colloid to exhibit self-propulsion, asymmetric forces must be continually present. A common microswimmer design strategy is to create solid colloids with permanent geometric or chemical asymmetry, i.e. Janus particles ${ }^{1,2}$. Localized chemical reactions occurring at the particle surface lead to gradient distributions of products that cause particle propulsion by mechanisms such as self-electrophoresis ${ }^{3}$, self-diffusiophoresis ${ }^{4,5}$, or bubble release $^{6}$. Isotropic liquid droplets can also self-propel via the Marangoni effect, but local chemical gradients must be maintained by a hydrodynamic instability ${ }^{7,8}$ or be externally applied ${ }^{9}$. Liquid Janus droplets, wherein the droplet is composed of two immiscible fluids each having an interface with the continuous phase, have been widely explored for uses such as particle templates ${ }^{10,11}$, optics ${ }^{12,13}$, and sensors ${ }^{14,15}$, but few examples of self-propelled Janus droplets have been described ${ }^{16-18}$. However, Janus droplets present exciting opportunities for control of chemotactic colloidal interactions, given the presence of multiple fluid phases that enable dynamic changes in chemical partitioning, and the tunability of droplet morphology via modification of droplet and surfactant chemistry. Here, we explore systematically how chemical composition, oil partitioning, droplet shape, and solubilization cooperatively influence the propulsion of Janus oil droplets in aqueous surfactant. By providing a deeper understanding of the principles and trends influencing Janus droplet swimming behaviors, we aim to demonstrate that such droplets can serve as a robust material platform for rational design of chemically-responsive, self-propulsive colloids.

The simplest droplet swimmers consist of a single dispersed fluid phase within a continuous phase, such as spherical oil droplets in water ${ }^{19,20}$ or water droplets in oil ${ }^{21}$. Active behavior is typically generated either by a reaction occurring at the droplet interface ${ }^{22}$ or by droplet solubilization into surfactant micelles ${ }^{23}$ that creates interfacial tension gradients and propels the drops via the Marangoni effect ${ }^{24}$. In the case of solubilization-driven propulsion, higher concentrations of solubilizate- "filled" micelles have been associated with higher interfacial tensions, causing droplets to move towards regions of solution containing more "empty" micelles and minimize interfacial energy ${ }^{25}$. Droplets can become self-propelled due to repulsion from their own solubilizate gradient when hydrodynamic instabilities result in spontaneous symmetry breaking of the fluid flows surrounding the droplet ${ }^{21,26}$. Overlap of chemical gradients resultant from nearby solubilizing droplets ${ }^{20,27,28}$, externally applied chemical gradients ${ }^{9,19}$, internal droplet asymmetries ${ }^{29}$, and interfacially adsorbed particles ${ }^{30}$ are also possible sources of chemical asymmetry that can induce droplet motion. Recently, we described how oil droplets exhibit predator-prey chasing interactions when placed in a system containing at least two droplet "species" of different chemical composition that act together in a source-sink framework ${ }^{31}$. A solubilizing predator droplet acts as an oil-filled micelle source, while nearby prey droplets uptake the predator's oil, acting as a sink. The prey's attenuation of the oil concentration attracts the predator drops while the prey drops are repulsed by the approaching predator, resulting in a 
chase. Inspired by the synergy resultant from the dynamic exchange of oils with different solubilization profiles and chemistry, and the inherent asymmetry that can be attained in a Janus colloid structure ${ }^{1}$, we sought to investigate the active behavior of solubilizing, biphasic Janus droplets.

In this work, we examine how the active swimming behaviors of Janus, biphasic oil-in-water droplets in surfactant are affected by droplet shape, droplet-internal oil partitioning, and oil solubilization kinetics. By characterizing Janus droplets containing many different combinations of oils in Triton X-100 surfactant, we elucidate the relationships between the degree of oil mixing, interfacial tensions, and oil solubilization rates and their effects on droplet propulsion. A key conclusion is that for droplets containing both a mobile (solubilizing) and non-mobile oil, the degree of partitioning of the mobile oil across the Janus droplets' oil-oil interface critically influences droplet speed and swimming direction. As a result, the propulsion speeds of Janus droplets containing an oil-oil interface can be over an order-of-magnitude faster compared to chasing pairs of single emulsion droplets of the same chemical composition and size. Tuning of the Janus droplet compartment volume ratios, surfactant concentration, and solution temperature allow further control over the Janus droplet propulsion. Spatiotemporal control over the swimming speed and orientation of droplets is demonstrated by applying thermal gradients induced via joule heating and laser spot illumination. We also explore interactions between Janus droplets, including the spontaneous formation of multi-droplet spinning clusters that rotate predictably based on cluster symmetry. Our findings provide insight as to how the chemistry and structure of multiphase droplets can be harnessed to design microswimmers with programmable self-propulsive and collective behaviors.

\section{Results and Discussion}

Comparison of chasing single emulsion droplets and Janus droplets. To investigate swimming Janus droplet behavior, we began by exploring Janus droplets containing a pair of oils previously known to exhibit active behavior through chasing interactions $r^{31}$ when present together as separate single emulsion droplets: 1-iododecane and ethoxynonafluorobutane (EFB) with drop diameters of approximately $60 \mu \mathrm{m}$ in $0.5 \mathrm{wt} \%$ Triton X-100 (Triton) nonionic surfactant (Figure 1a). Both oils sink in the aqueous solution lending to ease of experimentation. lododecane predator droplets readily undergo micellar solubilization (solubilization rate defined as change in droplet diameter $D$ over time $t,-d D / d t=0.22 \mu \mathrm{m} / \mathrm{min}$ ) while EFB prey droplets have negligible solubilization $(-d D / d t \approx 0 \mu \mathrm{m} / \mathrm{min})$. Once iododecane catches the EFB, the droplets form a pair that translates at speeds $(v)$ of up to $v=13 \mu \mathrm{m} / \mathrm{s}$ via the source-sink oil exchange framework previously described ${ }^{31}$ (Figure 1ai).

We prepared stable Janus droplets of iododecane and EFB in $0.5 \mathrm{wt} \%$ Triton using flow-focusing microfluidics, which allowed us to control the size of the droplets and the volume ratio of oils (Figure 1aii, Figure S1). The resultant shape of the droplets is governed by the balance of interfacial tensions ${ }^{32,33}$. We found that the iododecane-EFB Janus droplets dispersed in $0.5 \mathrm{wt} \%$ Triton swam in the same directional manner as the chasing droplet pair (i.e. iododecane in back, EFB in the front) but moved at speeds more than an order of magnitude faster, $v \approx 200 \mu \mathrm{m} / \mathrm{s}$ with long persistence lengths, many times the droplet length (Figure 1aii, Video S1). The Janus droplet speed gradually decreased over the droplet lifetime, which could be over an hour, as the iododecane tail of the droplet shrank and eventually disappeared due to solubilization (Figure 1b). Based on the observed droplet speeds and rate of volume change, the propulsion efficiency and Péclet number of the Janus droplet swimmer is estimated to be approximately 14 times greater than the chasing single emulsion droplets of the same chemistry and size (see Supporting Information sections, "Propulsion efficiency comparison" and "Péclet number calculation"). Higher surfactant concentrations correlated to faster swimming (Figure 1c). No droplet motion was observed at Triton concentrations below the critical micelle concentration (CMC) or when surfactant solution was presaturated with both oils, indicating that the droplet swimming mechanism depended on the kinetics of micelle-mediated oil solubilization. The interfacial tensions of the oils comprising the Janus droplet were higher in the presence of oil-filled Triton micelles as compared to empty micelles (Table S1), supporting the idea that droplets are propelled via the Marangoni effect towards solution containing less oil. When a second, fluorinated surfactant that solubilized the EFB was added, 1 wt $\%$ Capstone FS-30, no droplet motion was observed. Thus, preferential solubilization of one of the oils appeared important to the propulsion. The ratio of the Janus droplet compartment size (Figure S2a,b) and overall droplet size (Figure S2c) did influence the speeds, but all of these Janus droplets examined were still much faster than the chasing single emulsion droplet pair. 
As such, it appeared that the mere presence of the oil-oil interface was also a critical contributor to Janus drop swimming. An oil-oil interface would be expected to enable the direct partitioning of the oils between the Janus droplet compartments, whereas micelle-mediated oil transfer through the water is required for chasing single emulsion droplets to exchange oil. These different pathways for oil transport, we suspected, could be largely influential over the droplet swimming dynamics and required further investigation.

Influence of chemical composition and oil partitioning on Janus droplet propulsion speed and direction. To explore the influence of chemical partitioning across the oil-oil interface on the Janus droplet propulsion, we systematically varied the oil compositions and examined trends in droplet speed and shape (Figure 2). We note that when changing oil compositions, we are altering more than just chemical partitioning; the droplet shape can change due to alterations in the balance of interfacial tensions ${ }^{32,33}$, and oil solubilization rates ${ }^{34}$ - which provide the fuel for propulsion - can change as well. We attempt to consider these factors when designing the experiments and interpreting the results as described below.

We first examined Janus droplets containing iododecane paired with several other fluorinated oils of varying degrees of fluorination: methoxyperfluorobutane (MFB), 2-(trifluoromethyl)-3ethoxydodecafluorohexane (HFE-7500), perfluorohexane (PFH), perfluorooctane (PFO) and perfluorotributylamine (FC-43) (Figure 2). None of these fluorinated oils solubilize to a measurable extent in $0.5 \mathrm{wt} \%$ Triton $(-d D / d t \approx 0 \mu \mathrm{m} / \mathrm{min})$. These fluorinated oils have, respectively, a decreasing degree of partitioning, $x$, into iododecane, ranging from $8 \mathrm{vol} \%$ to $<0.1 \mathrm{vol} \%$, at room temperature as measured by changes in refractive index (Figure S3, Supporting Information "Oil partitioning measurement"). We define degree of oil partitioning $(x)$ such that fully miscible oils have $x=50 \mathrm{vol} \%$. We presume that the same partitioning trends also hold when considering the amount of iododecane in the fluorinated oil. All the fluorinated oil pairings with iododecane produced stable Janus droplets with similar shapes, where we define how "dumbbell-like" the droplet is by using the outer three phase contact angle; a perfectly spherical Janus drop we define to have a contact angle of $180^{\circ}$ and touching single emulsions have a contact angle of $0^{\circ}$. All iododecane Janus drops had contact angles between $110^{\circ}$ and $130^{\circ}$. All the Janus droplets swam in the same direction, with the solubilizing iododecane oil in the back (Figure 2a, yellow). However, the droplets swam at very different speeds depending on the non-solubilizing fluorinated oil; generally, the smaller the oil partitioning $x$, the slower the speed $v$. For instance, iododecane-EFB droplets swam at $v=$ $196 \pm 4 \mu \mathrm{m} / \mathrm{s}$ and had $x=7.9 \mathrm{vol} \%$, whereas iododecane-FC-43 droplets swam at $v=12 \pm 2 \mu \mathrm{m} / \mathrm{s}$ with $x$ $<0.1 \mathrm{vol} \%$. To correlate these trends with interfacial tensions, we measured the interfacial tensions of iododecane-saturated EFB, MFB, and FC-43 in $0.5 \mathrm{wt} \%$ Triton with and without iododecane-filled micelles. While the FC-43 had almost no difference in interfacial tension, the EFB showed an increase of $0.2 \mathrm{mN} / \mathrm{m}$ in the presence of oil-filled micelles (Table S1). The fact that the interfacial tension difference increased with the oil pair's $x$ is consistent with the observation that greater partitioning correlated with faster speeds.

When iododecane was replaced with the shorter chain length iodononane, droplet speeds increased overall (Figure 2a, orange), while replacement with longer chain length iodododecane led to reduced speeds (Figure 2a, blue). Most of the droplets had similar shapes (outer three-phase contact angles between $110^{\circ}$ and $130^{\circ}$ ). Partitioning of the fluorinated oils with the iodononane and iodododecane were similar to the iododecane ( $x=10.0 \mathrm{vol} \%, 6.7 \mathrm{vol} \%$, and $7.9 \mathrm{vol} \%$ for pairing with EFB, respectively) (Figure S3a). However, the solubilization rate of iodononane was much faster $(-d D / d t=0.34 \pm 0.03 \mu \mathrm{m} / \mathrm{min})$ and iodododecane much slower $(-d D / d t=0.09 \pm 0.01 \mu \mathrm{m} / \mathrm{min})$ than the iododecane $(-d D / d t=0.22 \pm 0.02$ $\mu \mathrm{m} / \mathrm{min}$ ) (Figure S4a). Thus, we conclude that when controlling for droplet shape and degree of partitioning, faster micelle-mediated solubilization led to faster Janus droplet swimming for mobile oils with similar chemical functionality.

When we changed the halogen atom on the haloalkane oil while maintaining carbon number (e.g. 1-chloro-, 1-bromo-, and 1-iododecane), we saw a greater diversity of droplet morphologies and a wider range of degrees of partitioning which corresponded to greater variation in the Janus droplet speeds (Figure 2b, Figure S3b). We take chlorodecane as the example to discuss the observed effects of droplet shape on the speed (Figure $\mathbf{2 b}$, grey). Chlorodecane and EFB are fully miscible, forming a single emulsion droplet that did not swim. Chlorodecane paired with MFB or HFE-7500 remained phase-separated but formed double or near-double emulsion droplets that swam slowly, if at all ( $v<5 \mu \mathrm{m} / \mathrm{s})$. The degree of partitioning in these two aforementioned oil pairs ( $x=13.6 \mathrm{vol} \%$ and $7.4 \mathrm{vol} \%$ respectively) is on par with that of iodononane paired with EFB or MFB ( $x=5.7$ vol\% and $10.0 \mathrm{vol} \%)$ and chlorodecane also solubilizes at a similar rate to iodononane $(-d D / d t=0.41 \mu \mathrm{m} / \mathrm{min}$ vs. $0.34 \mu \mathrm{m} / \mathrm{min})$. However, dumbbell-shaped 
iodononane-EFB and iodononane-MFB Janus droplets (contact angles between $110^{\circ}$ and $130^{\circ}$ ) swam an order of magnitude faster than the more nearly encapsulated chlorodecane-MFB and chlorodecane-HFE7500 drops $(v=191 \mu \mathrm{m} / \mathrm{s}$ and $268 \mu \mathrm{m} / \mathrm{s}$ vs. $<5 \mu \mathrm{m} / \mathrm{s}$ ), suggesting that the droplet shape asymmetry also plays a significant role in droplet speed. Chlorodecane paired with PFH and PFO formed more dumbbelllike droplets (contact angle $\approx 110^{\circ}$ ) and, correspondingly, swam significantly faster ( $v=159$ and $145 \mu \mathrm{m} / \mathrm{s}$ ). Chlorodecane paired with FC-43 formed a near-double emulsion drop shape and again swam more slowly $(v=43 \mu \mathrm{m} / \mathrm{s})$ and with shorter persistence lengths on the order of the droplet length, having both an unfavorable encapsulated drop shape as well as low degree of oil partitioning $(\approx 0.1 \mathrm{vol} \%)$. Thus, we conclude that an anisotropic Janus droplet shape in combination with a higher partitioning $(\sim 0.5 \mathrm{vol} \%$ or higher) and a faster rate solubilization $(-d D / d t>0.1 \mu \mathrm{m} / \mathrm{min})$ contributes to faster propulsion.

From the data presented in Figure $\mathbf{2 a} \mathbf{a}, \mathbf{b}$, it was clear that the oil partitioning $x$, oil solubilization rates, and droplet shape were all important factors governing the droplet speed $v$. However, these parameters are not independent, as they are all physical properties arising from the specific chemical interactions between the surfactants and oils. To empirically explore which parameter(s) might be the most directly useful in predicting droplet speeds, we examined the droplet speed as a function of each parameter (partitioning, solubilization rate, drop shape). The only parameter that exhibited direct correlation with droplet speed was the oil degree of partitioning (Figure S5). The data for each haloalkane was fitted empirically using a function with the form: $v=a * x * e^{-x / b}$, where $v$ is the swim speed, $x$ is the degree of partitioning, and $a$ and $b$ are the fitting parameters which we propose are related to solubilization rate and the sensitivity of the interfacial tensions to solubilized oil (Figure S5). Using these empirical fits we then plotted drop speed (normalized to the peak speed $v_{\max }$ as fitted for each haloalkane) versus the partitioning $x$ (normalized to $x$ associated with $v_{\max }$ for each haloalkane) for each Janus droplet in Figure 2a,b. We find all Janus droplets fit well to $v=x * e^{-x}$ (Figure 2c). Thus, for a given mobile oil, the partitioning of the mobile and non-mobile oil inside the Janus drop appears to be a good predictor of the droplet speed.

For all oil combinations in Figure 2, the Janus droplets swam with the mobile, solubilizing haloalkane in the back and the non-mobile fluorinated oil in the front. However, when we examined Janus droplets containing a non-mobile fluorinated silicone polymer (25\%-35\% nonafluorohexylmethylsiloxane, $65-75 \%$ dimethyl siloxane copolymer, 8-12 cP) paired with mobile dibromooctane $(-d D / d t=0.65 \mu \mathrm{m} / \mathrm{min})$, we noticed anomalous behavior; upon adding these droplets to fresh $0.5 \mathrm{wt} \%$ Triton, they initially propelled with the dibromooctane in back at $v>60 \mu \mathrm{m} / \mathrm{s}$, but after tens of seconds, slowed to a halt then swam in the reverse orientation at $v=13 \mu \mathrm{m} / \mathrm{s}$ (Video S2, Figure 3a). The droplets remained swimming in this 'reverse' orientation until all the dibromooctane had been solubilized. Reversal in the swimming orientation was also observed for dibromooctane paired with $100 \mathrm{cP}$ and 10,000 cP polydimethylsiloxane. The reversal in swimming orientation suggests that the oil solubilization profile of the droplets changes as a function of time to reach a different steady state condition than the 'forward' swimmers. Since the non-mobile oil compartment in the reverse swimmers is higher viscosity than the forward swimmers, potentially, the mobile oil is not able to continuously repartition fast enough during droplet swimming to maintain a critical steady state concentration of mobile oil and solubilization rate on both sides of the droplet necessary for forward motion. Variation in the concentration profiles of oil-filled micelles across the droplets surface caused by changes in their internal distribution of oils could lead to differences in the interfacial tension gradients across the droplet surface and different propulsion direction.

To compare the forward and reverse orientation swimmers, we used particle image velocimetry (PIV) to map the external flows (Figure 3b, Video S3). Results revealed distinct Marangoni force-generated flow profiles before and after direction reversal that suggest differences between the swimming mechanisms. The majority of the flow in the plane of movement surrounding the forward Janus swimmer were emitted parallel to the direction of motion. The reverse swimmer, however, had its fastest flows in the directions perpendicular to droplet motion, which do not contribute towards propulsion, and also carried a small eddy of flow at the rear of the droplet. The more prominent perpendicular flows and correspondingly slower speeds for the reverse swimmers compared to their forward swimming counterparts suggest that the solubilization profile maintained by the reverse swimmers less efficiently harnesses directional Marangoni flows for propulsion because of the limited ability to repartition the mobile oil between drop compartments. In both swimming cases, the planar flow field we measure shows a net flow extending from the droplet, indicating a contribution of non-visualized fluid from the area above the droplets as they move along the dish bottom (seen also in Video S8). 
Proposed Janus droplet swimming mechanism as influenced by oil partitioning, droplet shape, and solubilization rates. Based on the observed correlations between droplet shape, solubilization rates, oil partitioning, and droplet speed described in Figures 1-3, we propose the following generalized framework for rationalizing Janus droplet swimming behaviors (Figure 4). Janus droplet motion is driven by Marangoni flows resultant from an asymmetric distribution of a mobile (solubilizing) oil across the droplet surface. Higher concentrations of solubilized oil correspond to higher interfacial tensions resultant from oilsurfactant interactions ${ }^{25}$. To minimize surface energy, droplets propel towards regions with lower concentrations of solubilized oil and more "free" surfactant. While this general propulsion mechanism is similar to that of solubilizing single emulsion droplets, there are several additional considerations for swimming Janus droplets.

In a Janus droplet, there are two oils present; depending on which surfactant is used in the continuous phase, these two oils can be solubilized at different rates such that they can be either both mobile, both non-mobile, or one mobile and one non-mobile; we generally define a mobile oil has having $d D / d t>0.1 \mu \mathrm{m} / \mathrm{min}$ and non-mobile as $-d D / d t<<0.1 \mu \mathrm{m} / \mathrm{min}$, and we presume that the mobile oil generates an interfacial tension gradient upon solubilization. If both oils are non-mobile, the droplet will not swim via a solubilization-driven mechanism, so we do not consider this case further. The oils can also have different degrees of partitioning within each other, $x$, governing the concentrations of each oil within each compartment of the Janus droplets as they swim. We expect $x$ to affect the rate of solubilization of the mobile oil across each compartment's interface resulting in differences in the gradient profile of mobile oil surrounding each droplet, and hence different interfacial tension gradients. Further, the oils can assume different geometries within the droplet as governed by the balance of interfacial tensions between liquids $^{32,33}$. The droplet shape also affects the gradient profile of the mobile oil released by solubilization. Thus, we assert the asymmetries arising during steady-state droplet motion result from: 1) the physical asymmetry of the droplet shape (determined by three-phase contact angle and droplet size), and 2) the chemical asymmetry of the mobile oil gradient produced by mobile oil solubilization from both drop compartments (determined by partitioning $x$ and solubilization rate $-d D / d t$ ). We conceptually consider each of these combinations of factors below in four general cases, depicted in Figure 4 . We note that these four cases are simplified and meant to provide principles to guide the design of active Janus droplets with control over speed, persistence, and directionality. Future work, including development of fluid mechanical models, would be necessary to fully understand how the droplet shapes, solubilization kinetics, interfacial tension gradients, and non-equilibrium oil partitioning balance to govern the Janus droplet swimming dynamics.

Case 1. A nearly encapsulated Janus droplet (or double emulsion) containing at least one mobile oil. For a droplet in which one oil is fully or near fully encapsulated by the other, the gradient of solubilized oil is largely symmetric. This case is more comparable to a self-propelled single emulsion droplet having high symmetry such that spontaneous symmetry breaking and hydrodynamic instabilities are necessary to maintain motion. This case is exemplified by chlorodecane-MFB, chlorodecane-HFE-7500, bromodecaneEFB or chlorodecane-FC-43 droplets in Figure 2.

Case 2. An anisotropic Janus droplet with similar micelle-mediated solubilization from both compartments. If micelle-mediated solubilization is occurring uniformly around the entire Janus droplet, the interfacial tension gradients will be minimized and so the droplet may move slowly or not move at all. We observed this case for a Janus droplet containing two mobile oils: iododecane-EFB droplets in a surfactant mixture of Capstone FS-30 and Triton. We imagine that this case could also occur if the mobile oil partitioning into the non-mobile oil was very high such that the mobile oil was solubilizing at similar rates across the entire droplet surface resulting in a symmetrical distribution of oil-filled micelles.

Case 3. An anisotropic Janus droplet containing mobile and non-mobile oil compartments with negligible partitioning. In this case, the mobile oil solubilizes nearly exclusively from only one compartment of the Janus droplet during stead-state swimming, and the droplet will propel with the mobile oil compartment in the front, non-mobile oil compartment in the back, in order to maximize the advection of surfactant solution containing more empty micelles to the droplet interface (Figure 4b). This case is exemplified by the dibromooctane-fluorosilicone droplets in Triton in Figure 3, wherein we propose that the reversal in droplet orientation is due to the fact that the mobile dibromooctane cannot continually repartition into the fluorosilicone as quickly as it is solubilized from the fluorosilicone during swimming; this leads to the eventual depletion of the mobile oil within the non-mobile oil compartment and the swimming direction reversal. This case also likely describes the swimming of Janus droplets containing non-solubilizing silicone polymer and solubilizing liquid crystal oils as reported in the literature ${ }^{17}$. 
Case 4. An anisotropic Janus droplet containing mobile and non-mobile oils with some degree of oil partitioning. The mobile oil having finite solubility in the non-mobile oil compartment $(x>0.1 \mathrm{vol} \%)$ can partition across the oil-oil interface and solubilize along the entire surface of the Janus droplet. Since the mobile oil is present in different concentrations within the drop compartments, we expect that the mobile oil may solubilize at different rates from each compartment (Figure 4c) ${ }^{34}$. An extended, anisotropic gradient of solubilized oil across the length of the entire droplet is generated resulting in larger interfacial tension differences along the droplet surface achieving faster Marangoni propulsion than Case 3 droplets, selfpropelled single emulsion droplets, and chasing single emulsion pairs under equivalent surfactant concentrations. As the droplet swims, the mobile oil redistributes across the oil-oil interface to maintain a steady state concentration and continuous solubilization across the length of the droplet. The majority of the droplets in Figure 1 and Figure 2 exemplifies this situation.

Harnessing thermal gradients to manipulate Janus droplet dynamics. Following from our proposed framework, we expect that application of stimuli which modify oil partitioning, solubilization rates, and droplet shapes can be used to manipulate Janus droplet swimming behaviors. Higher temperatures, for instance, are expected to accelerate the kinetics of oil solubilization ${ }^{34}$ as well as favor mixing between the haloalkanes and fluorinated oils ${ }^{35}$. To examine how temperature would affect droplet swimming, we used a temperature regulated microscope chamber and measured the swimming speed of iododecaneEFB Janus drops in $0.5 \mathrm{wt} \%$ Triton between $10^{\circ} \mathrm{C}$ and $50{ }^{\circ} \mathrm{C}$. As the temperature was increased from 10 ${ }^{\circ} \mathrm{C}$ to $30^{\circ} \mathrm{C}$, Janus droplet speed steadily increased, as did the iododecane solubilization rate as evidenced by faster shrinkage of the droplet volume (Figure 5a). However, at temperatures above $30{ }^{\circ} \mathrm{C}$, the Janus droplet shape changed, and the droplet speed slowed, even though iododecane was still solubilizing. We attribute this speed reduction to the more spherical droplet shape (i.e. "Case 1"); it is also possible that the iododecane may be solubilizing at similar rates from both sides of the Janus droplet due to higher partitioning at these elevated temperatures (i.e. "Case 4") thereby reducing the asymmetry in the oil gradient, although this is difficult to experimentally probe. By substituting EFB with HFE-7500, a more highly fluorinated oil that has less favorable mixing with the iododecane, the maximum swimming speed could be raised above $420 \mu \mathrm{m} / \mathrm{sec}$ at temperatures between $40^{\circ} \mathrm{C}$ and $50^{\circ} \mathrm{C}$, and the droplets did not become more spherical at higher temperatures.

To examine the effect of thermal gradients as a possible method to control droplet swimming behavior, we used a microscale Joule heater under different applied voltages (Figure S6) and used an IR camera to map the thermal profile as a function of distance from the electrodes (Figure $\mathbf{5 b}$, bottom plot). Droplets of iododecane-EFB droplets in $0.5 \mathrm{wt} \%$ Triton with $0.1 \mathrm{M} \mathrm{NaCl}$ were added $10 \mathrm{~mm}$ away from the heater and their positions with corresponding speeds were analyzed (Figure $\mathbf{5 b}$, top plot). Under the application of a voltage, droplets swam towards the heater electrodes and accelerated as they approached, directionally propelling up the thermal gradient even when the gradient was minimal, $<0.5^{\circ} \mathrm{C} / \mathrm{mm}$. Control experiments conducted with 75-90 $\mu \mathrm{m}$ polyethylene tracer particles $\left(1.02 \mathrm{~g} / \mathrm{cm}^{3}\right)$ and single emulsion droplets composed of either iododecane and EFB oils produced significantly reduced motion $(<20 \mu \mathrm{m} / \mathrm{s}$ within $500 \mu \mathrm{m}$ of heater under the highest voltage), ruling out effects of heating-driven convection and thermocapillary motion as primary causes of the observed acceleration of Janus droplets as they approached the heater.

After establishing that thermal gradients are effective stimuli for control of droplet speed and orientation, we sought to manipulate the Janus droplets with a more easily patternable heat source: a continuous wave near-infrared laser $(780 \mathrm{~nm})$ focused through the bottom of the sample chamber. When the laser was focused in a fixed position, randomly swimming iododecane-EFB Janus droplets in $0.5 \mathrm{wt} \%$ Triton gathered around the laser spot over the course of a few minutes in a radial fashion with the EFB compartment closer to the laser (Figure 5c, Video S4). As the size of the assembled droplet cluster grew, an area depleted of droplets surrounding the laser beam began to form (Figure 5c, rightmost panel). We attribute this depletion zone to convective flows which draw oil-saturated surfactant solution along the dish bottom towards the center of the droplet cluster, imparting a repulsive force that balances the attraction of droplets towards the higher temperature center. By scanning the laser beam across collections of droplets, we could direct the droplet orientation, although the orientation remained stable only for several minutes after the beam had passed (Figure 5d, Video S5).

Janus droplet interactions and collective behaviors. Solubilizing droplets not only respond to self-generated gradients, but they can also respond to the chemical gradients produced by droplet 
neighbors, leading to multibody and collective interactions ${ }^{27,36}$. As shown previously in Figure 1, isolated Janus droplets of iododecane-EFB in $0.5 \mathrm{wt} \%$ Triton swam in a linear fashion with a stable speed of around $200 \mu \mathrm{m} / \mathrm{s}$. However, when the number density of droplets was increased such that droplets were forced to swim through regions of solution recently occupied by another droplet, a slowdown in speed accompanied by a slight reorientation in direction often occurred (Figure 6a,b, Video S6). This slowdown is caused by the "exhaust trail" ${ }^{37}$ of solubilized oil in micelles left behind as the droplets swim. As the chemical trail ages and oil-filled micelles diffuse, intersecting droplets experienced a smaller drop in speed as they crossed through the trail region (Figure 6b,c). We tracked and analyzed droplet speeds during more than 200 trail interaction events to generate a relative speed decrease vs. trail age dataset; when fitted to an exponential recovery model, this data gave a relaxation timescale in good agreement with the diffusivity of the Triton micelles $^{38}$ over the droplet trail width approximated using the Stokes-Einstein equation (Figure 6c, Supporting Information section "Analysis of droplet trail interactions").

Collective effects on active behavior due to multi-body droplet interactions were also observed when larger numbers of Janus droplets (hundreds to thousands) were added to the sample chamber leading to high local droplet number density. For example, Janus droplets of iododecane-MFB in $0.5 \mathrm{wt} \%$ Triton spontaneously formed coordinated clusters of three to five droplets which rotated at up to 28 revolutions per minute (rpm) (Figure $6 \mathbf{d}$, Video S7). The rotating assemblies were stable for minutes and rotated in either clockwise or counter-clockwise directions dependent on the cluster symmetry. Reverse swimmers composed of dibromooctane-fluorosilicone in $0.5 \mathrm{wt} \%$ Triton also formed spinning clusters but the orientation of the Janus droplets within the spinner was flipped compared to the forward swimmers such that the solubilizing dibromooctane oil was closer to the cluster center (Figure 6e). Visualization of the external flows in the plane directly above spinning droplet assemblies using tracer particles revealed steady inward motion of fluid, while flows in the droplets plane were ejected outwards away from the cluster (Figure 6f, Video S8). These observations suggest that the formation of spinning assemblies may be favored due to a stable vortex of fresh surfactant solution being pumped downward by the cooperative rotating motion. If the Janus droplets were prevented from moving, such as by affixing iododecane-EFB Janus droplets to a glass coverslip by surface wetting, droplet pumps were generated that directionally moved fluid from the front of the droplet to the back (Video S9). Overall, control over the arrangement of droplets through their collective swimming interactions or the manipulation of thermal profiles may be used to spatially organize and harness the pumping behavior ${ }^{39}$ of droplets.

\section{Conclusions}

We have shown that Janus oil droplets undergoing micellar solubilization in surfactant solutions can exhibit a rich diversity of controllable, active behaviors. Through a systematic investigation of oil chemistry and partitioning, droplet shape, surfactant conditions, and temperature, we have begun to elucidate the underlying drivers and key design principles that govern Janus droplet motion. Our experimental results point to the importance of oil partitioning between the Janus droplet compartments in determining droplet swimming speed and direction. We demonstrated many combinations of swimming Janus droplets containing haloalkane, fluorocarbon, and silicone oils, with speeds reaching over several hundred microns per second, and provide chemical design principles for tuning of the Janus droplet dynamics. Collective interactions between droplets, such as spinning droplet cluster formation and chemical trail interactions, were observed and analyzed. Further studies aimed at understanding and modeling the pathways and kinetics of solubilization in multiphase droplets will lead to additional insights for controlling droplet dynamics and multibody effects. We believe that active Janus droplets may be used to test and inspire new models for the collective dynamics of interacting microswimmers ${ }^{40,41}$ and serve as the foundation for chemical design of more complex materials with emergent active behaviors ${ }^{42-44}$.

\section{Acknowledgments}

LZ acknowledges funding from the Army Research Office grant W911NF-18-1-0414 and the Marion Milligan Mason Award for Women in the Chemical Sciences. AS acknowledges funding by the Department of Energy, Office of Basic Energy Sciences (DOE-DE-SC0020964). PM acknowledges funding by Department of Energy Biomolecular Materials (DE-SC0010426)

\section{References}


1 Jurado-Sanchez, B., Pacheco, M., Maria-Hormigos, R. \& Escarpa, A. Perspectives on Janus micromotors: Materials and applications. Applied Materials Today 9, 407-418 (2017).

2 Paxton, W. F. et al. Catalytic nanomotors: autonomous movement of striped nanorods. Journal of the American Chemical Society 126, 13424-13431 (2004).

3 Wong, F., Dey, K. K. \& Sen, A. Synthetic micro/nanomotors and pumps: fabrication and applications. Annual Review of Materials Research 46, 407-432, doi:10.1146/annurev-matsci070115-032047 (2016).

4 Moran, J. L. \& Posner, J. D. Phoretic self-propulsion. Annual Review of Fluid Mechanics 49, 511540 (2017).

5 Anderson, J. L. Colloid transport by interfacial forces. Annual Review of Fluid Mechanics 21, 6199 (1989).

6 Gao, W., Uygun, A. \& Wang, J. Hydrogen-bubble-propelled zinc-based microrockets in strongly acidic media. Journal of the American Chemical Society 134, 897-900 (2012).

7 Maass, C. C., Krüger, C., Herminghaus, S. \& Bahr, C. Swimming Droplets. Annual Review of Condensed Matter Physics 7, 171-193, doi:10.1146/annurev-conmatphys-031115-011517 (2016).

8 Schmitt, M. \& Stark, H. Swimming active droplet: A theoretical analysis. EPL (Europhysics Letters) 101, 44008 (2013).

9 Lagzi, I., Soh, S., Wesson, P. J., Browne, K. P. \& Grzybowski, B. A. Maze solving by chemotactic droplets. Journal of the American Chemical Society 132, 1198-1199 (2010).

10 Lone, S. \& Cheong, I. W. Fabrication of polymeric Janus particles by droplet microfluidics. RsC Advances 4, 13322-13333 (2014).

11 Frank, B. D., Antonietti, M. \& Zeininger, L. Structurally Anisotropic Janus Particles with Tunable Amphiphilicity via Polymerization of Dynamic Complex Emulsions. Macromolecules (2020).

12 Goodling, A. E. et al. Colouration by total internal reflection and interference at microscale concave interfaces. Nature 566, 523-527, doi:10.1038/s41586-019-0946-4 (2019).

13 Nagelberg, S. et al. Reconfigurable and responsive droplet-based compound micro-lenses. Nature Communications 8 (2017).

14 Balaj, R. V. \& Zarzar, L. D. Reconfigurable complex emulsions: Design, properties, and applications. Chemical Physics Reviews 1, 011301 (2020).

15 Zarzar, L. D., Kalow, J. A., He, X., Walish, J. J. \& Swager, T. M. Optical visualization and quantification of enzyme activity using dynamic droplet lenses. Proceedings of the National Academy of Sciences 114, 3821-3825, doi:10.1073/pnas.1618807114 (2017).

16 Li, M., Brinkmann, M., Pagonabarraga, I., Seemann, R. \& Fleury, J.-B. Spatiotemporal control of cargo delivery performed by programmable self-propelled Janus droplets. Communications Physics 1, 23, doi:10.1038/s42005-018-0025-4 (2018).

17 Jeong, J. et al. Liquid crystal Janus emulsion droplets: preparation, tumbling, and swimming. Soft Matter 11, 6747-6754 (2015).

18 Li, M. et al. Kinetics of active water/ethanol Janus droplets. Soft Matter 16, 6803-6811 (2020).

19 Jin, C., Krüger, C. \& Maass, C. C. Chemotaxis and autochemotaxis of self-propelling droplet swimmers. Proceedings of the National Academy of Sciences 114, 5089-5094 (2017).

20 Moerman, P. G. et al. Solute-mediated interactions between active droplets. Physical Review $E$ 96, 032607 (2017).

21 Izri, Z., van der Linden, M. N., Michelin, S. \& Dauchot, O. Self-Propulsion of Pure Water Droplets by Spontaneous Marangoni-Stress-Driven Motion. Physical Review Letters 113, 248302 (2014).

22 Hanczyc, M. M., Toyota, T., Ikegami, T., Packard, N. \& Sugawara, T. Fatty acid chemistry at the oil- water interface: self-propelled oil droplets. Journal of the American Chemical Society 129, 9386-9391 (2007). 
Christian, S. D. \& Scamehorn, J. F. Solubilization in surfactant aggregates. Vol. 55 (CRC Press, 1995).

24 Saville, D. The effects of interfacial tension gradients on the motion of drops and bubbles. The Chemical Engineering Journal 5, 251-259 (1973).

25 Herminghaus, S. et al. Interfacial mechanisms in active emulsions. Soft Matter 10, 7008-7022 (2014).

26 Guyon, E., Hulin, J.-P., Petit, L. \& Mitescu, C. D. Physical hydrodynamics. (Oxford university press, 2001).

27 Hokmabad, B. V., Saha, S., Canalejo, J. A., Golestanian, R. \& Maass, C. C. Quantitative characterization of chemorepulsive alignment-induced interactions in active emulsions. arXiv preprint arXiv:2012.05170 (2020).

Soto, R. \& Golestanian, R. Self-Assembly of Catalytically Active Colloidal Molecules: Tailoring Activity Through Surface Chemistry. Physical Review Letters 112, 068301-068306, doi:10.1103/PhysRevLett.112.068301 (2014). Hokmabad, B. V., Baldwin, K. A., Krüger, C., Bahr, C. \& Maass, C. C. Topological stabilization and dynamics of self-propelling nematic shells. Physical Review Letters 123, 178003 (2019). Cheon, S. I., Batista Capaverde Silva, L., Khair, A. \& Zarzar, L. Interfacially-Adsorbed Particles Enhance the Self-Propulsion of Oil Droplets in Aqueous Surfactant. (2020).

31 Meredith, C. H. et al. Predator-prey interactions between droplets driven by non-reciprocal oil exchange. Nature Chemistry 12, 1136-1142 (2020).

32 Guzowski, J., Korczyk, P. M., Jakiela, S. \& Garstecki, P. The structure and stability of multiple micro-droplets. Soft Matter 8, 7269-7278 (2012).

33 Zarzar, L. D. et al. Dynamically reconfigurable complex emulsions via tunable interfacial tensions. Nature 518, 520-524, doi:10.1038/nature14168 (2015).

34 Carroll, B. J. The kinetics of solubilization of nonpolar oils by nonionic surfactant solutions. Journal of Colloid and Interface Science 79, 126-135 (1981).

35 Gladysz, J. A., Curran, D. P. \& Horváth, I. T. Handbook of Fluorous Chemistry. (John Wiley \& Sons, 2006).

36 Lippera, K., Benzaquen, M. \& Michelin, S. Alignment and scattering of colliding active droplets. Soft Matter (2020).

37 Izzet, A. et al. Tunable Persistent Random Walk in Swimming Droplets. Physical Review X 10, 021035 (2020).

38 Paradies, H. H. Shape and size of a nonionic surfactant micelle. Triton X-100 in aqueous solution. The Journal of Physical Chemistry 84, 599-607 (1980).

$39 \mathrm{Yu}, \mathrm{T}$. et al. Microchannels with Self-Pumping Walls. ACS Nano, doi:10.1021/acsnano.0c05826 (2020).

40 Thutupalli, S., Geyer, D., Singh, R., Adhikari, R. \& Stone, H. A. Flow-induced phase separation of active particles is controlled by boundary conditions. Proceedings of the National Academy of Sciences 115, 5403-5408 (2018).

41 Krüger, C., Bahr, C., Herminghaus, S. \& Maass, C. C. Dimensionality matters in the collective behaviour of active emulsions. The European Physical Journal E 39, 64 (2016).

42 Lach, S., Yoon, S. M. \& Grzybowski, B. A. Tactic, reactive, and functional droplets outside of equilibrium. Chemical Society Reviews 45, 4766-4796 (2016).

43 Ross, T. D. et al. Controlling organization and forces in active matter through optically defined boundaries. Nature 572, 224-229 (2019).

44 Gentile, K., Somasundar, A., Bhide, A. \& Sen, A. Chemically Powered Synthetic "Living" Systems. Chem 6, 2174-2185 (2020). 


\section{Figures}
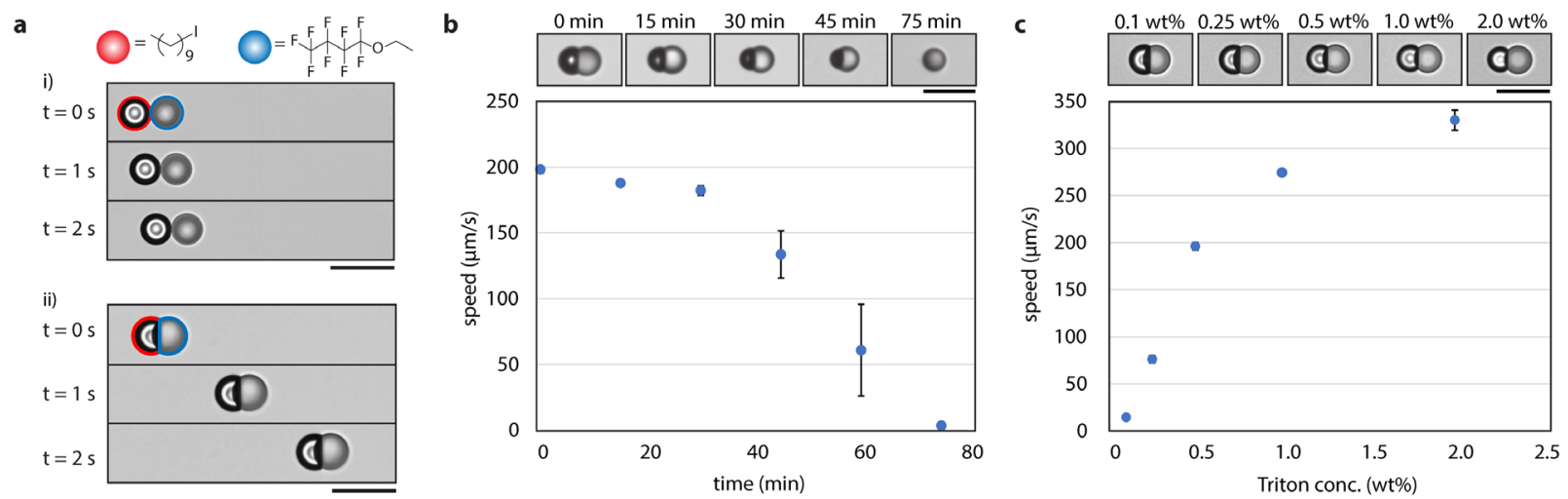

Figure 1: lododecane and EFB oils paired in a Janus droplet exhibit faster speeds than chasing single emulsion droplets and speeds are dependent on oil compartment size and surfactant concentration. a) Time sequence micrographs comparing: (i) the speed of chasing iododecane (red) and EFB (blue) single emulsion droplets, and (ii) a Janus droplet of the same oils in $0.5 \mathrm{wt} \%$ Triton. Scale, 100 $\mu \mathrm{m}$. b) Janus droplets composed of iododecane and EFB oils in $0.5 \mathrm{wt} \%$ Triton show a gradual decline in speed over time as iododecane is solubilized. Optical micrographs of Janus droplets at varying timepoints are shown. Scale, $100 \mu \mathrm{m}$. c) The speeds of iododecane and EFB Janus droplets vary as a function of Triton surfactant concentration. Optical micrographs of Janus droplets at different Triton concentrations are shown. Scale, $100 \mu \mathrm{m}$. 

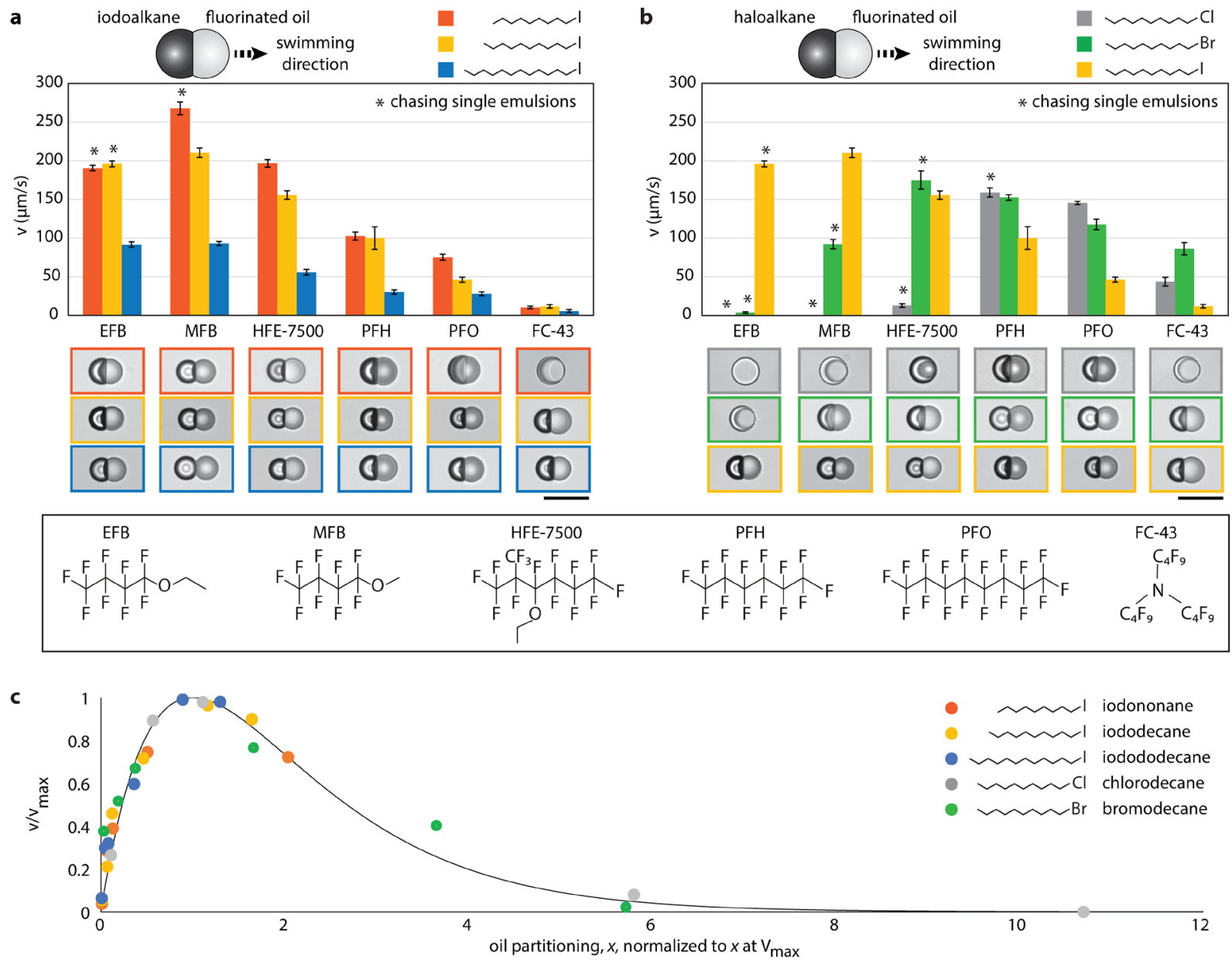

Figure 2: The partitioning of oils within the Janus droplet and the droplet shape influence the swimming speed. For both (a) and (b), Janus droplets composed of varying haloalkane and fluorinated oils were made in $0.5 \mathrm{wt} \%$ Triton, and their speeds and droplet shapes were analyzed. In (a), speeds of Janus droplets composed of iodoalkane oils of varying chain lengths each paired with several fluorinated oils were compared. In (b), speeds of Janus droplets composed of 1-chloro-, 1-bromo, and 1-iododecane each paired with several fluorinated oils were compared. The ordering of the fluorinated oils along the $x$ axis from left to right follows the trend of decreasing partitioning of the haloalkane into the fluorinated oil (Figure S3). An asterisk ( $\left.{ }^{*}\right)$ indicates oil pairings which exhibit single emulsion droplet chasing. Each data point represents the average value of five speed measurements obtained from the displacements of different droplets over a 5 second period with standard deviation error bars. Optical micrographs of the Janus droplets formed from each oil pairing are aligned beneath each plot. The colored border of the micrographs indicates the haloalkane, and the column indicates the fluorinated oil. The Janus droplet images are oriented with the haloalkane is the left-side compartment and the fluorinated oil is the right-side. If swimming, droplets move rightward. EFB and chlorodecane are miscible. Chemical structures of the fluorinated oils are given at bottom. Scale, $100 \mu \mathrm{m}$. (c) The normalized speeds $v / v_{\max }$ of each haloalkane paired with the fluorinated oils are plotted as a function of the pairs' partitioning $x$ normalized to the $x$ associated with $v_{\max }$ for each haloalkane. The fit curve is the function $y=x^{*} e^{1-x}$. The chlorodecane-EFB droplet was omitted from the plot because these oils are miscible. 

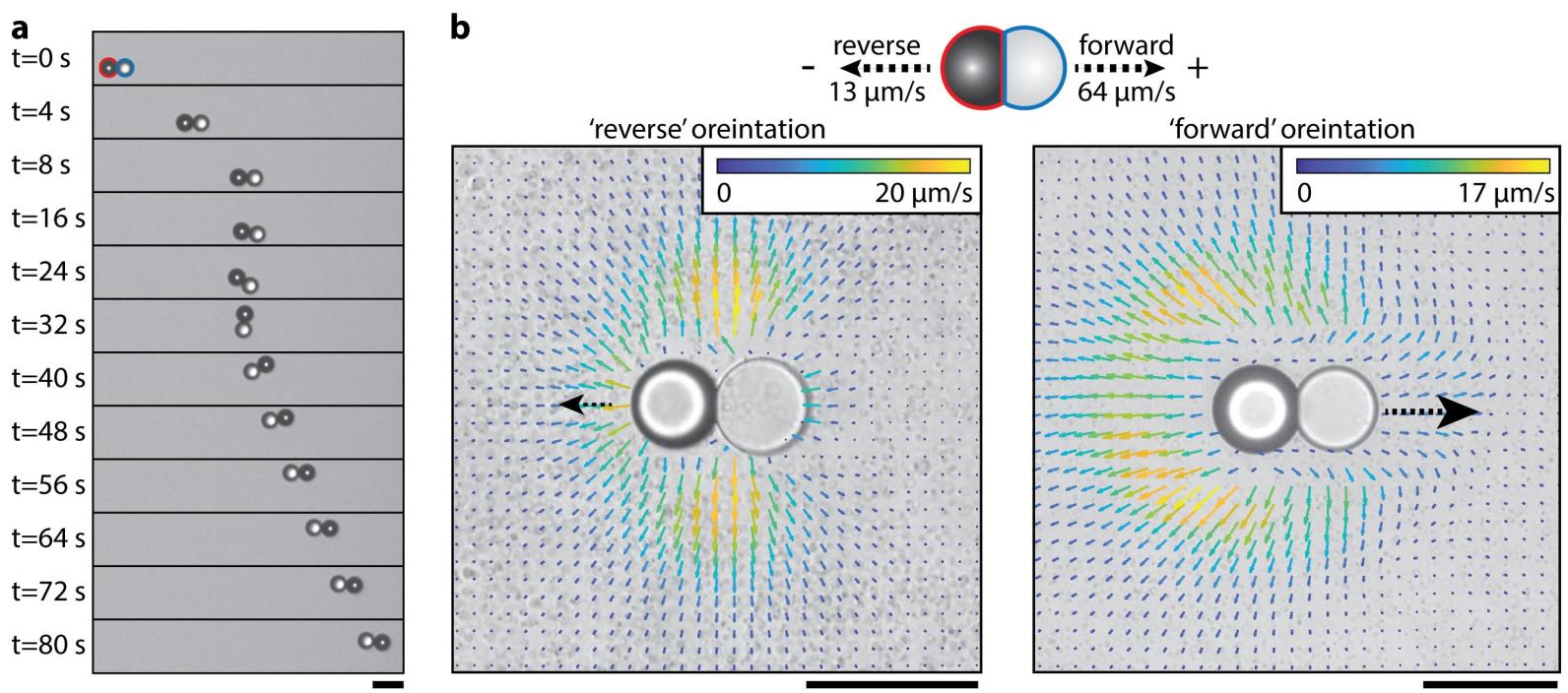

Figure 3: Janus droplet swimming orientation can reverse over time. (a) Time sequence micrographs showing the swimming behavior of dibromooctane (red) and fluorosilicone (blue) Janus droplets in $0.5 \mathrm{wt} \%$ Triton. Initially, the droplet moves with dibromooctane in the back, fluorosilicone in the front (i.e. a 'forward' swimmer), but after a matter of seconds, the drop reverses orientation and moves with dibromooctane in front (i.e. 'reverse' swimmer). The droplet persists in this reverse orientation for the duration of its lifetime. See Video S2. Scale, $100 \mu \mathrm{m}$. (b) The external flow profiles surrounding a Janus droplet composed of dibromorooctane-fluorosilicone with 'forward' and 'reverse' swimming orientations in $0.5 \mathrm{wt} \%$ Triton solution visualized using PIV analysis of suspended tracer particles. See Video S3. Scale, $100 \mu \mathrm{m}$. 

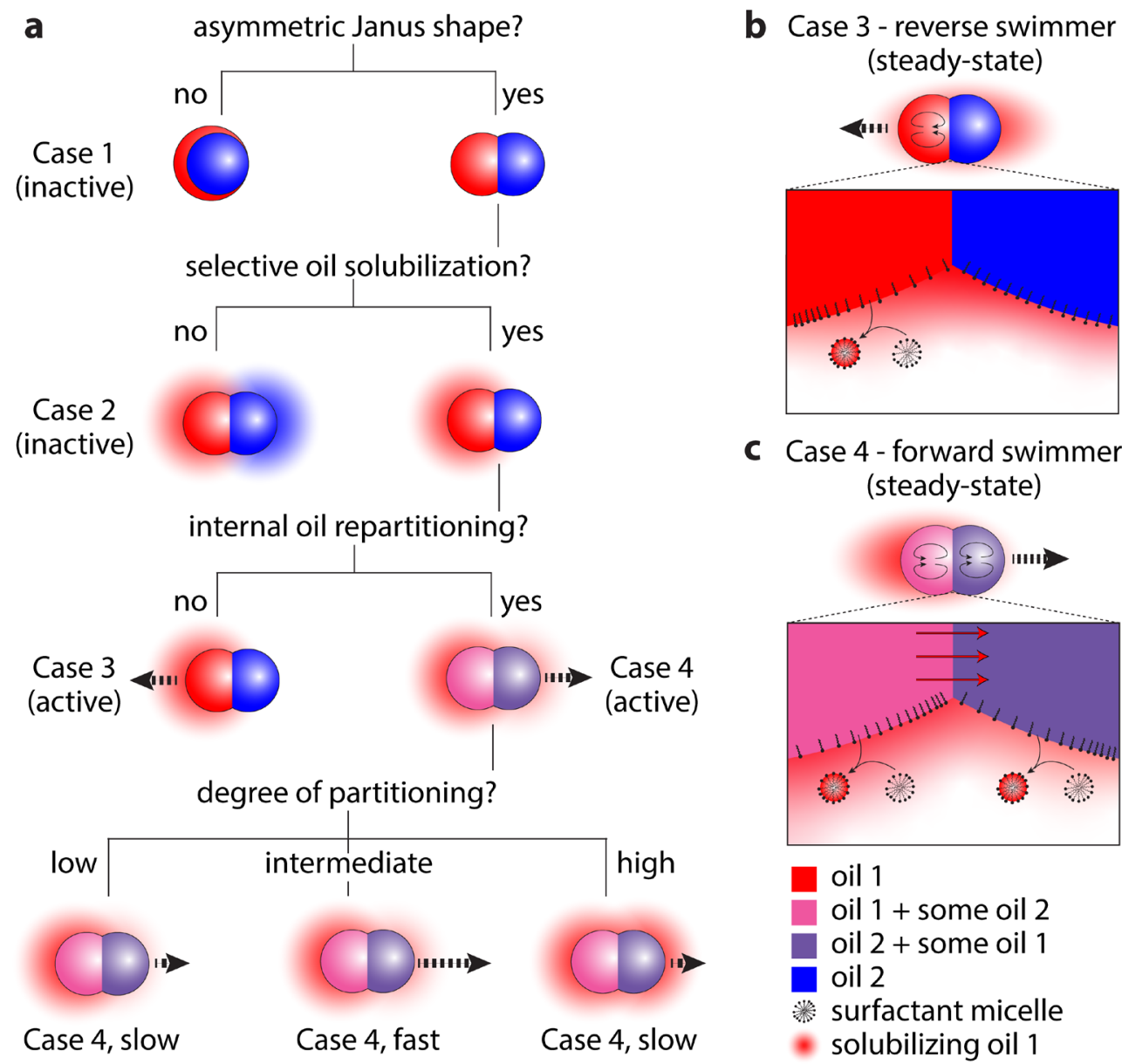

c Case 4 - forward swimmer (steady-state)

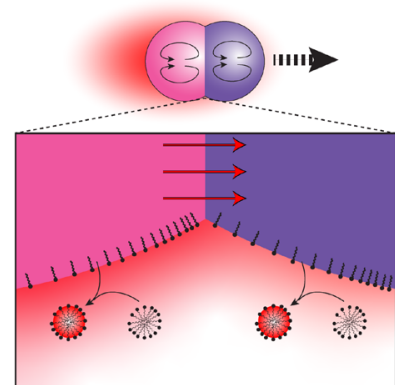

oil 1

oil $1+$ some oil 2

oil $2+$ some oil 1

oil 2

* surfactant micelle

solubilizing oil 1

Figure 4: Balance between solubilization, oil partitioning across the Janus droplet's internal interface, and droplet shape affects swimming behaviors. (a) A decision tree relating the swimming behaviors of four general droplet "cases" we have observed experimentally. Each droplet contains two oils (oil 1 and oil 2) in different geometries, with different degrees of mutual partitioning, and different micellemediated solubilization as depicted in the diagrams. Case 1: the droplet is spherical, e.g., a double or neardouble emulsion, where oil 1 nearly fully encapsulates oil 2 and the solubilized oil gradient is largely symmetric. We find that such droplets tend to swim slowly, if at all. Case 2: an anisotropic Janus droplet is formed but both oil 1 and oil 2 are mobile. We find these droplets to swim slowly, if at all. Case 3: an anisotropic Janus droplet is formed, but the mobile oil 1 is only solubilizing from one compartment. This case could arise because the oils do not partition into each other, or the rate of replenishment of oil 1 into the oil 2 compartment occurs slowly compared to loss by solubilization such that a significant steady-state concentration of oil 1 inside the oil compartment is not maintainable. We find that such droplets swim with the solubilizing oil in the front (i.e. the 'reverse' swimmers). Case 4: an anisotropic Janus droplet is formed, and the oils have partitioning so as to maintain a swimming steady-state concentration of oil 1 inside the oil 2 compartment, and only oil 1 is solubilizing across the entire droplet surface. The droplet swims with oil 1 in the back and oil 2 in the front (i.e. 'forward' swimmers). Depending on the degree of oil partitioning we find Case 4 droplets to exhibit a range of swim speeds with the fastest speeds observed for intermediate partitioning values (Figure 2 and Figure S5). (b,c) Schematic illustrations at the droplet and molecular scale for forward and reverse type swimming droplets are shown. We depict the proposed directions of internal Marangoni flows, oil solubilization gradients, interfacial tension gradients, and internal oil transport. 
a

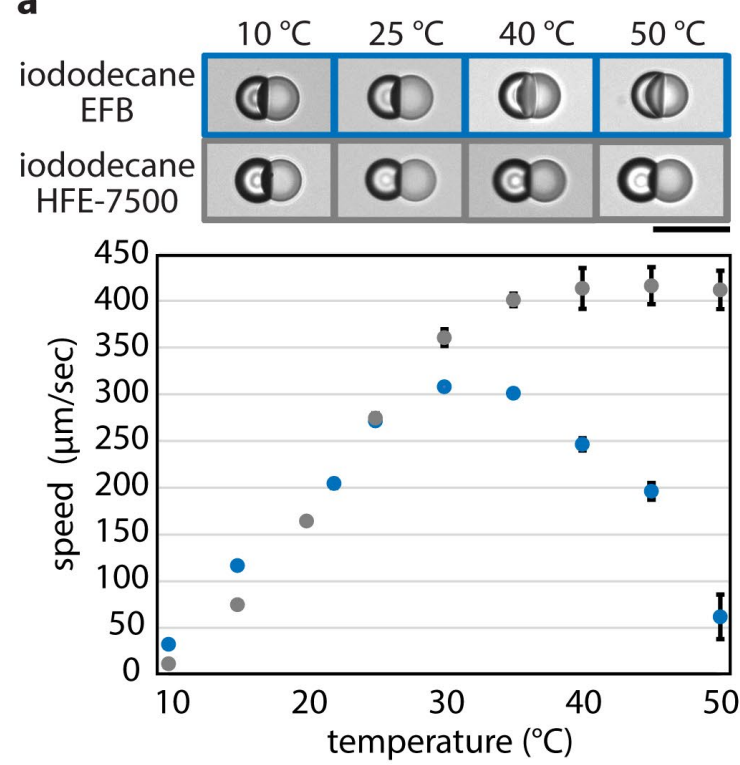

b

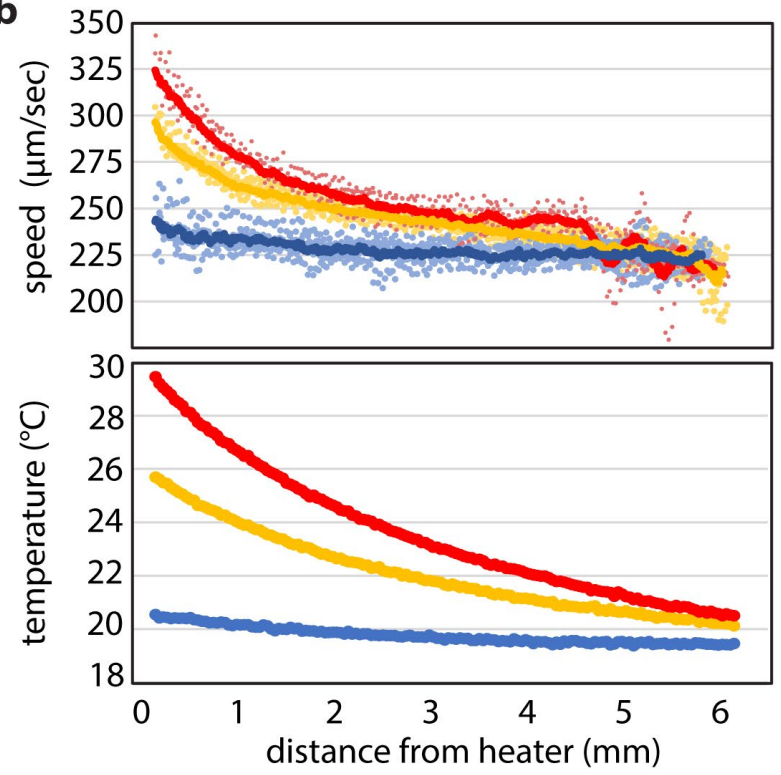

c laser spot on: $t=0 \mathrm{~s}$

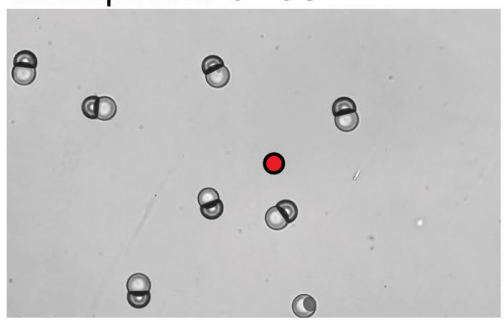

d laser scanning on: $t=0 \mathrm{~s}$

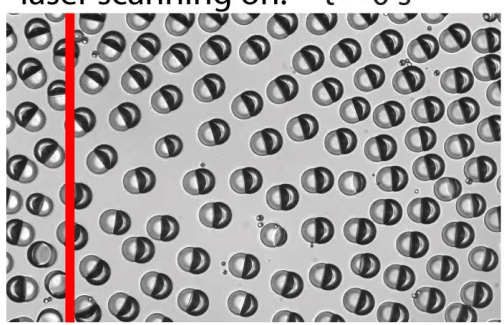

$t=23 s$

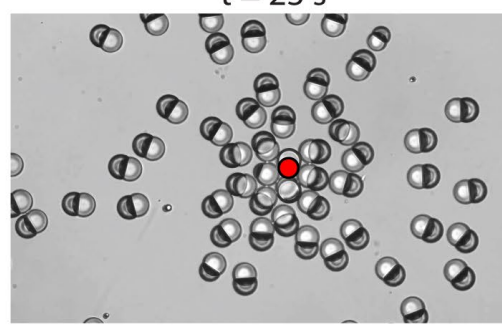

$\mathrm{t}=26 \mathrm{~s}$

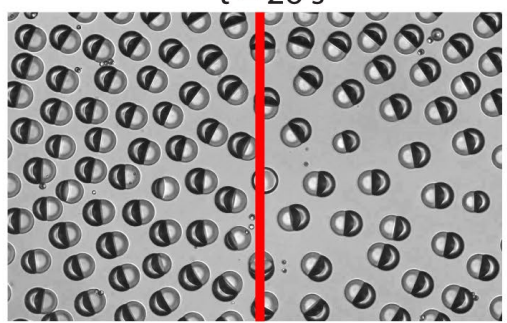

$\mathrm{t}=77 \mathrm{~s}$

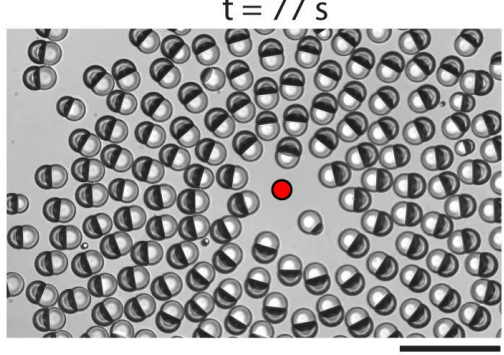

$\mathrm{t}=41 \mathrm{~s}$

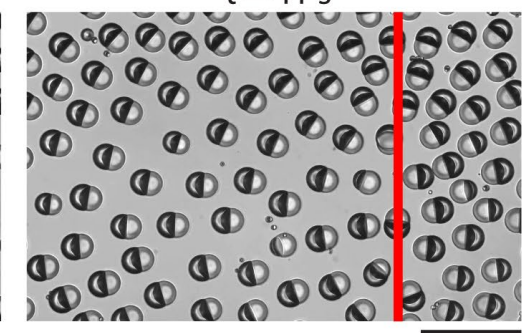

Figure 5: Thermal gradients are used to control Janus droplet speeds, positioning, and organization. (a) The shapes and speeds of iododecane-EFB and iododecane-HFE-7500 Janus droplets in $0.5 \mathrm{wt} \%$ Triton were analyzed at temperatures between $10^{\circ} \mathrm{C}$ and $50^{\circ} \mathrm{C}$. Each data point represents the average of five speed measurements obtained from the displacements of different droplets over a 5 second period with standard deviation error bars. Optical micrographs of droplets at different temperatures are given. Scale, $100 \mu \mathrm{m}$. (b) A microscale Joule heater was used to apply a thermal gradient to iododecaneEFB droplets in $0.5 \mathrm{wt} \%$ Triton and $0.1 \mathrm{M} \mathrm{NaCl}$ solution. See Figure $\mathbf{S 6}$ for a diagram of the heater design. Janus droplets were added $10 \mathrm{~mm}$ away from the heater electrodes and the droplet speeds were analyzed as a function of distance from the heater (top plot). Data points represent the instantaneous tracked speeds of five different droplets swimming towards the heater at each voltage, while the line represents the moving average determined by the nearest 10 data points. An IR camera was used to determine the solution temperature as a function of distance from the heater electrodes (bottom plot). (c) lododecane-EFB Janus droplets in $0.5 \mathrm{wt} \%$ Triton were exposed to a $780 \mathrm{~nm}$ laser at $250 \mathrm{~mW}$, focused at the red dot in each optical micrograph. The laser was left "on" and over time the solution heated locally, attracting droplets that organized radially. The EFB side of the Janus droplet always pointed towards the laser. See Video S4. Scale, $250 \mu \mathrm{m}$. (d) lododecane-EFB Janus droplets in $0.5 \mathrm{wt} \%$ Triton were exposed to a scanning $780 \mathrm{~nm}$ 
laser. The laser was scanned back and forth along the red line at a rate of 0.2 pass/second. As the laser line was passed across the field of view, the droplets reoriented themselves such that the EFB side of the droplet always faced towards the laser. See Video S5. Scale, $250 \mu \mathrm{m}$. 
a

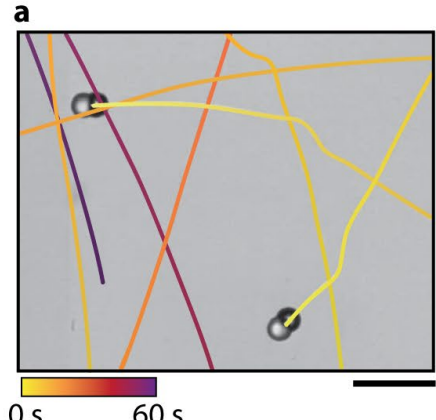

b

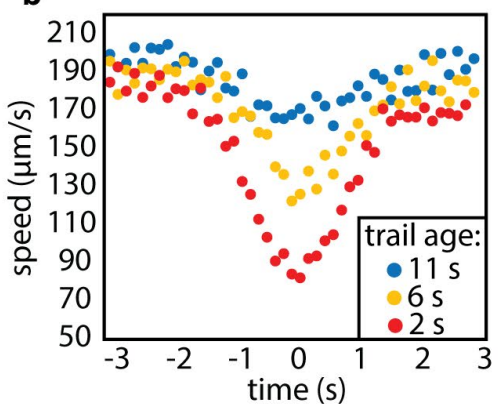

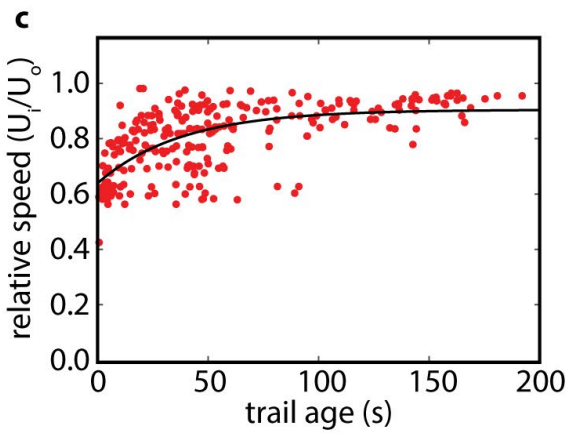

d

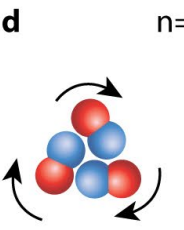

$n=$

3
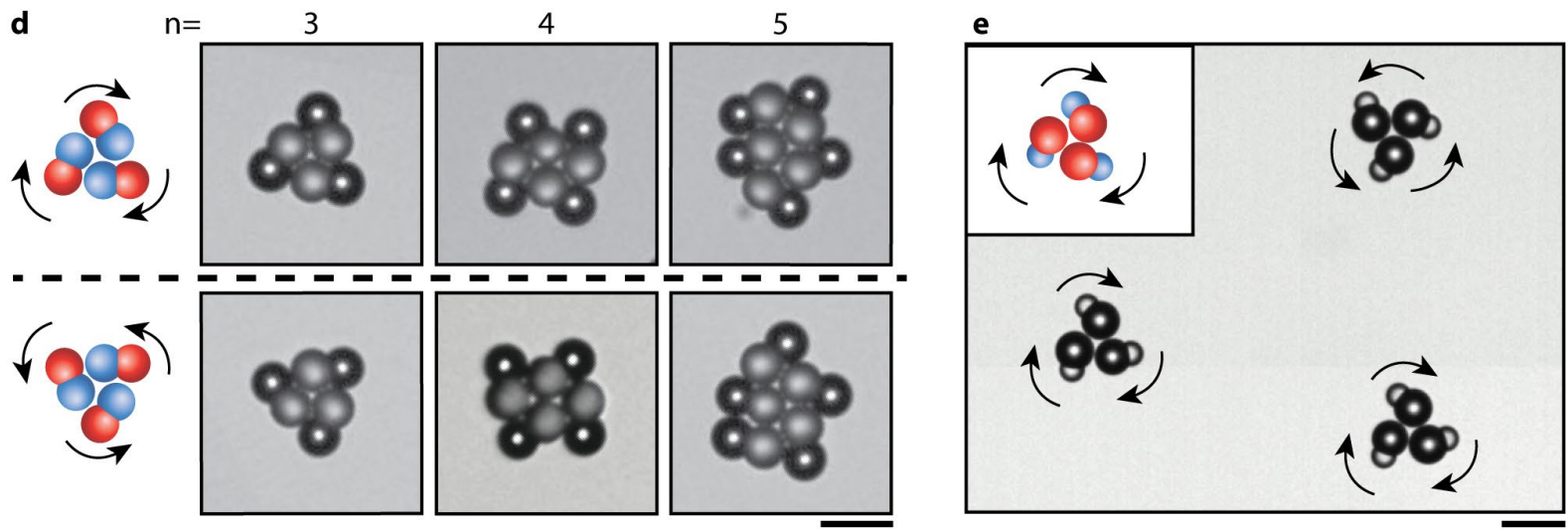

f
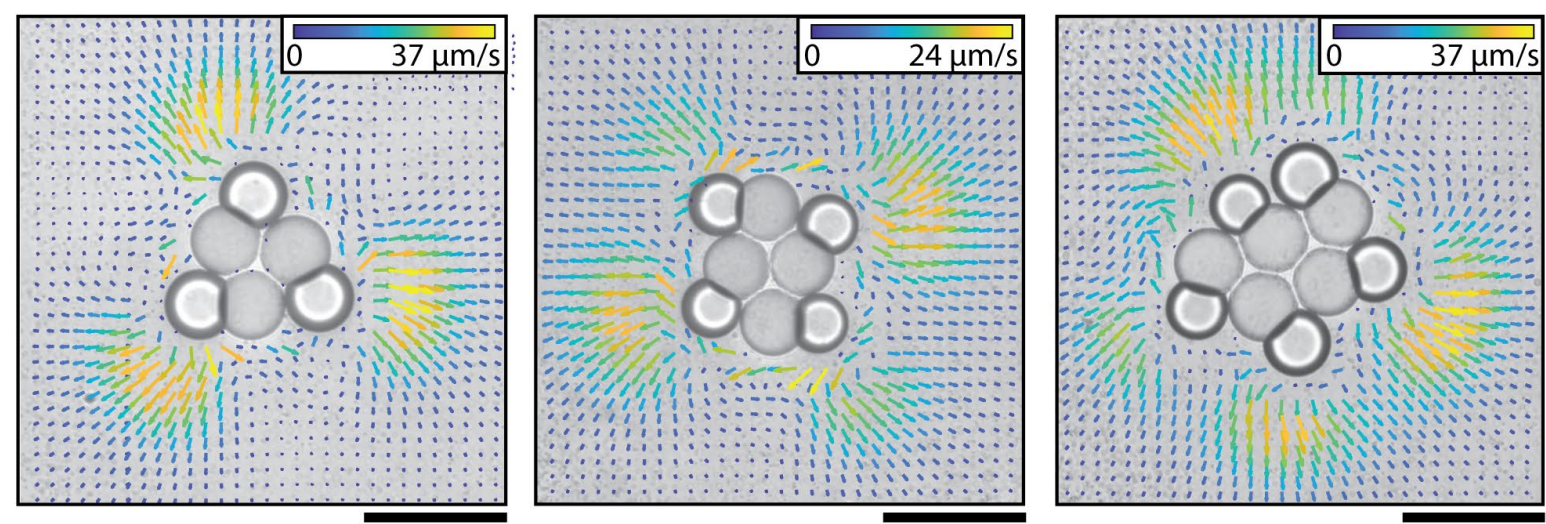

Figure 6: Multi-body interactions between Janus droplets. (a) An optical micrograph shows iododecane-EFB Janus droplets in $0.5 \mathrm{wt} \%$ Triton with colored traces of the droplet trails over 60 seconds. Trails are color coded by lifetime according to the color scale. When droplets swim over a recent trail, they experienced a disturbance in their movement leading to a slight swerving of direction and slowdown in speed (Video S6). Scale, $250 \mu \mathrm{m}$. (b) The speeds of individual iododecane-EFB Janus droplets were analyzed as they crossed over trails of solubilized oil left by other droplets while swimming in $0.5 \mathrm{wt} \%$ Triton. Data points represent the instantaneous speeds of three separate droplets while crossing over trails with ages of 2, 6, and 11 seconds. The timepoint of trail crossing was defined as zero seconds. (c) The minimum speed of individual iododecane-EFB Janus droplets in $0.5 \mathrm{wt} \%$ Triton as they crossed a trail of solubilized oil were analyzed and plotted as a function of trail age. Speed values are presented as a relative fraction of the initial speed before and after the crossing event, obtained using a custom Matlab tracking tool. The black line represents the fitting of an exponential recovery model based on the scaling of the magnitude of speed decrease with the time lag between interactions. (d) Assemblies of $n=3,4$, or 5 Janus droplets composed of iododecane (red) and methoxyperfluorobutane (blue) in $0.5 \mathrm{wt} \%$ Triton spin directionally based on the assembly symmetry. Assemblies of $n=3$ rotate at $22 \pm 5 \mathrm{rpm}, \mathrm{n}=4$ rotate at $18 \pm 4 \mathrm{rpm}$, and of $n=5$ rotate at $15 \pm 3 \mathrm{rpm}$ as determined from the average and standard deviation of five isolated droplet clusters measured over one minute. See Video S7. Scale, $100 \mu \mathrm{m}$. (e) Reverse swimmers composed of 
dibromooctane (red) and fluorosilicone (blue) form assemblies that spin as well, but the solubilizing dibromooctane is at the cluster center. Arrows indicate spinning direction. Scale, $100 \mu \mathrm{m}$. (f) External flow profiles surrounding spinning clusters of iododecane-methoxyperfluorobutane Janus drops in $0.5 \mathrm{wt} \%$ Triton obtained using PIV tracking of suspended tracer particles. Visualizing the external flows revealed the stability of droplet assemblies is sustained by a vortex of surfactant solution being pumped downward onto the cluster (Video S8). Scale, $100 \mu \mathrm{m}$. 


\section{Supplementary Information for}

\section{Chemical design of self-propelled Janus droplets}

Caleb H. Meredith ${ }^{1}$, Alexander Castonguay², Yu-Jen Chiu', Allan M. Brooks ${ }^{3}$, Pepijn Moerman ${ }^{4}$, Peter Torab $^{5}$, Pak Kin Wong, ${ }^{5,6,7}$, Ayusman Sen ${ }^{2,3}$, Darrell Velegol ${ }^{3}$, Lauren D. Zarzar ${ }^{1,2,8^{*}}$

1. Department of Materials Science and Engineering, The Pennsylvania State University, University Park, PA 16802, USA

2. Department of Chemistry, The Pennsylvania State University, University Park, PA 16802, USA

3. Department of Chemical Engineering, The Pennsylvania State University, University Park, PA 16802, USA

4. Department of Chemical and Biomolecular Engineering, Johns Hopkins University, Baltimore, MD 21218, USA

5. Department of Mechanical Engineering, The Pennsylvania State University, University Park, PA 16802, USA

6. Department of Biomedical Engineering, The Pennsylvania State University, University Park, PA 16802, USA

7. Department of Surgery, College of Medicine, The Pennsylvania State University, Hershey, PA 17033, USA

8. Materials Research Institute, The Pennsylvania State University, University Park, PA 16802, USA

Lauren D. Zarzar

Email: Idz4@psu.edu

\section{This PDF file includes:}

Supplementary text

Figures S1 to S6

Table S1

Legends for Videos S1 to S9

SI References

Other supplementary materials for this manuscript include the following:

Videos S1 to S9 


\section{Supplementary Information Text}

Chemicals and materials. Capstone FS-30, 1-(ethoxy) nonafluorobutanes (mixture of $n$ - and isobutyl isomers) and perfluorohexane(s) (98\%), perfluorotributylamine (FC-43) (Synquest Laboratories); 2(trifluoromethyl)-3-ethoxydodecafluorohexane (HFE-7500), perfluorooctane (PFO) (TMC Industries); methoxyperfluorobutane (mixture of $n$ - and isobutyl isomers) (99\%), Triton X-100, 1-chlorodecane (98\%), 1-iodononane (copper stabilized, 98\%), silicone oil (10,000 cSt) (Sigma Aldrich); 1,8-dibromooctane (98\%), 1-bromodecane (98\%), 1-iododecane (copper stabilized, 98\%), and 1-iodododecane (copper stabilized, 98\%) (Alfa Aesar); polydimethylsiloxane, polydimethylsiloxane trimethylsiloxy terminated (5970 MW, $100 \mathrm{cSt}),(25-35 \%$ nonafluorohexylmethylsiloxane, 65-75\% dimethylsiloxane) copolymer (8-12 cSt) (Gelest, Inc.); polybead carboxylate microspheres (0.97 $\mu \mathrm{m}$ diameter, latex) (Polysciences, Inc.); polyethylene microspheres $\left(75-90 \mu \mathrm{m}, 1.02 \mathrm{~g} / \mathrm{cm}^{3}\right)$ (Cospheric). All chemicals were used as received without further preparation except for copper stabilized iodoalkane oils which were filtered prior to droplet fabrication using a nylon syringe filter $(0.20 \mu \mathrm{m}$ pore size) (Millipore).

Droplet fabrication. The fabrication of monodisperse emulsion droplets was carried out using a fourchannel flow focusing hydrophilic glass chip with a channel diameter of $100 \mu \mathrm{m}$ (Dolomite). Each microchannel inlet was connected to a reservoir of the desired liquid. To form Janus droplets, the inlets of the inner two channels were connected to reservoirs of different pairs of immiscible oils (for example iododecane and EFB) with polyether ether ketone tubing of inner diameter 0.0025 inch $(63.5 \mu \mathrm{m})$, outer diameter 1/16 inch $(1.59 \mathrm{~mm})$ and a length of 20 inches $(50.8 \mathrm{~cm})$, and the outer two fluid phases that contained aqueous surfactant solution (for example, $0.5 \mathrm{wt} \%$ Triton) were connected to the reservoirs with polyether ether ketone tubing of inner diameter 0.005 inch $(127 \mu \mathrm{m})$, outer diameter 1/16 inch $(1.59$ $\mathrm{mm}$ ) and length 20 inches $(50.8 \mathrm{~cm}$ ) (Figure S1). The flow rates of each liquid were manipulated with a Fluigent MFSC-EZ pressure controller to control the volume ratio of each oil compartment and droplet size. Typical pressures used for the inner phase fluids ranged from 1.0 to 4.0 bar, and typical pressures used for the outer phase fluids ranged from 0.5 to 6.0 bar. Droplets suspended in surfactant solution were collected from the microfluidic device outlet in $2 \mathrm{~mL}$ plastic storage vials until experiments for use within 24 hours.

Protocol to observe Janus droplet individual and multibody droplet swimming behavior. To observe and analyze Janus droplet swimming behavior, a glass-bottomed dish of 1.5 inch $(3.8 \mathrm{~cm})$ diameter with aluminum walls was filled with approximately $1 \mathrm{~mL}$ of surfactant solution and then approximately 30-50 droplets were transferred from a storage vial via a micropipette to the center of the dish forming a loose packed droplet cluster about 0.5 to $1.0 \mathrm{~mm}$ wide. After dispensing the droplets, the dish was covered with a clear lid and undisturbed for the remainder of the experiment. Janus droplets became active immediately after settling to the glass bottom of the dish and aligning on their sides (long axis of the droplet parallel to the plane of the dish) swimming in different directions away from the cluster starting location. Average speed values for were obtained by measuring linear displacements over period of at least 5 seconds of droplets swimming radially in different directions away from the cluster. To setup experiments with spinning droplet assemblies, a larger number of droplets (approximately $300-1000$ ) was pipetted into the dish forming a high number density area (as seen in Video S7). The spinning assemblies formed spontaneously. Droplet swimming persisted until the mobile oil within the Janus droplet had been completely solubilized (typically on the order of an hour). Droplet lifetime could be extended by choosing oils that solubilized more slowly, starting with larger volume droplets, or using a less concentrated surfactant solution. Droplet motion was not observed when concentrations of surfactant below the critical micelle concentration were used. To test droplet swimming in oil-saturated $0.5 \mathrm{wt} \%$ Triton X-00, droplets were allowed to equilibrate in $2 \mathrm{~mL}$ of surfactant solution for more than a week. Droplets did not swim when placed in the imaging dish containing the oil-saturated surfactant supernatant. Chasing single emulsion droplet pair interactions were observed using a similar procedure as described in previous work ${ }^{1}$.

Droplet imaging. Droplet interactions were observed using an inverted optical microscope (Nikon, Eclipse Ti-U) in brightfield transmission mode between $\times 2$ and $\times 40$ magnification. Images were recorded using an attached camera (Andor, Zyla 4.2P sCMOS) in video mode with a range from 0.1 to 20 frames per second. 
Oil solubilization rate measurement. Oil solubilization rates, defined as the change in droplet diameter over time, were obtained by measuring the shrinkage rate of isolated individual oil droplets (starting diameter, $60-100 \mu \mathrm{m}$ ) in different surfactant solutions at room temperature over a period of at least 20 minutes, in triplicate. Time-lapse images were analyzed with calibrated pixel values using the Nikon Elements-D software suite. The rate of droplet diameter change was found to be constant over the observation period, which indicates an interfacially limited solubilization process, consistent with previous kinetic solubilization studies on nonionic surfactants ${ }^{2}$. Solubilizing droplets smaller than $40 \mu \mathrm{m}$ often became self-propelled, as characterized by their persistent directional motion in the absence of other surrounding droplets.

Oil partitioning measurement. The partitioning degrees of fluorinated and haloalkane oils used to form Janus droplets were analyzed based on refractive index measurements performed using a temperaturecontrolled J457FC refractometer (Rudolph Research) at $20^{\circ} \mathrm{C}$. The degree of partitioning between pairs of oils was determined by comparing the measured refractive indices of each pure oil to the refractive index of the haloalkane oil saturated with the fluorinated oil. The percent miscibility in terms of volume fraction of partitioned oil was quantified by taking the difference between the refractive index of the pure haloalkane oil and the fluorinated-oil-saturated haloalkane oil divided by the difference between the refractive index of the two pure oils. Using changes in refractive index to estimate degree of oil mixing seems reasonable based on experiments with fully miscible pairs of haloalkane and fluorinated oils in which we found that the relative degree of refractive index change was linear as a function of the volume fraction of each oil ${ }^{1}$. Saturation of the haloalkane oils was achieved by adding $0.5 \mathrm{~mL}$ of each oil to a plastic vial, shaking the two liquids and allowing them to equilibrate into two phase separated layers for at least 30 minutes before measurement. A micropipette was used to extract $200 \mu \mathrm{L}$ of the haloalkane-rich phase which was then deposited directly onto the refractometer sensor before promptly covering the sample with a lid to limit evaporation. A single measurement for each oil was recorded up to four decimal places after the instrument obtained a stable index value, typically within 10 seconds. Using this method, a fully miscible oil pair is defined to have a partitioning of $50 \%$. The refractive index of the haloalkane-rich phase was measured instead of the fluorinated oil-rich phase mixtures due to the higher vapor pressures of the fluorinated oils ${ }^{3}$, which sometimes produced continued drift in refractive index values over time as a result of evaporation of the sample.

Propulsion efficiency comparison. The propulsion efficiency comparison for an iododecane-EFB Janus droplet swimmer and chasing pair of iododecane and EFB single emulsion droplets of similar size in 0.5 wt $\%$ Triton was estimated based off the observed speeds of each droplet type $(198 \mu \mathrm{m} / \mathrm{s}$ and $13 \mu \mathrm{m} / \mathrm{s}$ respectively) and volume loss of iododecane fuel due to solubilization. A solubilization rate of $0.22 \mu \mathrm{m} / \mathrm{min}$ was determined for single emulsions of iododecane in $0.5 \mathrm{wt} \%$ Triton, using the protocol described in the "Oil solubilization rate measurement" section. The volume of iododecane solubilized during Janus droplet swimming was calculated using the first two images from time sequence in Figure 1b, assuming the iododecane compartment was a spherical cap attached the EFB side of the droplet $(78.3 \mathrm{pL}$ and $60.4 \mathrm{pL}$ at $0 \mathrm{~min}$ and $15 \mathrm{~min}$, respectively); the total loss of iododecane oil due to solubilization over the 15 minute Janus droplet swimming period was thus estimated to be $17.9 \mathrm{pL}$. Given the same starting diameter (59 $\mu \mathrm{m})$ and linear solubilization rate of an iododecane single emulsion drop $(0.22 \mu \mathrm{m} / \mathrm{min})$, the oil volume loss over the same 15 minute time period for a single emulsion droplet could be estimated (16.5 pL). By comparing the speeds and iododecane volume loss between the swimming Janus droplet and chasing pair of single emulsions, we find the Janus drop to be approximately 14 times more efficient:

$$
\frac{198 \mu \mathrm{m} / \mathrm{s}}{17.9 \mathrm{pL}} \approx 14 * \frac{13 \mu \mathrm{m} / \mathrm{s}}{16.5 \mathrm{pL}}
$$

Péclet number calculation. We define the relevant Péclet number as the ratio between the rate of advective and diffusive transport of oil. We calculated the Péclet number for swimming droplets swimming using the equation:

$$
P e=v * l / D
$$


where $P e$ represents the dimensionless Péclet number, $v$ is equal to droplet speed, I represents the radius of Janus droplet compartments, and $D$ the diffusion coefficient for oil-filled Triton micelles. Given a droplet radius of $30 \mu \mathrm{m}$ and diffusion coefficient of $4.910^{-11} \mathrm{~m}^{2} / \mathrm{s},{ }^{4}$ a chasing pair of iododecane-EFB droplets moving at $13 \mu \mathrm{m} / \mathrm{s}$ would have a Péclet number of 8 while a Janus droplet of equivalent size moving at $198 \mu \mathrm{m} / \mathrm{s}$ would have a Péclet number of 121.

Analysis of droplet external flows. The external flows surrounding swimming Janus droplets were visualized using latex microspheres as tracer particles suspended in $0.5 \mathrm{wt} \%$ Triton surfactant solution (Videos S3 and S8). Janus droplets prepared using microfluidics in the absence of tracer particles were added to a dish containing the surfactant solution with $1 \mu \mathrm{m}$ tracer particles following the procedure described in "Protocol to observe Janus droplet individual and multibody droplet swimming behavior". Videos of the tracer particle movement were recorded at a rate of 20 frames per second. To analyze the flow profiles around moving droplets, the position of each droplet in the video was first tracked using custom tracking code written in Matlab 2019b and based on the code of Crocker and Grier ${ }^{5}$. Then the flow profiles were calculated using PIVlab, a Particle Image Velocimetry extension tool for Matlab ${ }^{6}$. A mask was placed over the droplets in each frame using their tracked positions in order to only measure flow of fluid surrounding the droplets and not the droplets themselves. The flow velocity was calculated using the FFT based multipass algorithm in 4 passes, with square interrogation areas with length 256, 128,64, and 32 pixels respectively. In each pass, the step length was half the length of the interrogation area. The flow profiles of multiple frames within one video were averaged by rotating and translating each profile such that the droplet centers coincide (Figure 3b, Figure 6f).

Measurement of the droplet swimming speed versus bulk temperature changes. The swimming speeds of Janus droplets as a function of temperature were measured using a temperature regulated microscope chamber (mK2000, INSTEC) by adjusting and fixing the temperature of $0.5 \mathrm{wt} \%$ Triton surfactant solutions between $10{ }^{\circ} \mathrm{C}$ and $50{ }^{\circ} \mathrm{C}$ at $5{ }^{\circ} \mathrm{C}$ intervals. To conduct experiments, $1 \mathrm{~mL}$ of surfactant solution was added to the same aluminum-walled glass bottom dish described for previous speed measurements and placed into the chamber for 5 minutes with the lid sealed to allow for temperature equilibration. After removing the lid, 30-50 droplets were quickly added to the center of the dish via micropipette before reclosing the chamber. The droplet speed values in Figure 5a are the average of least five droplets measured using the same procedure described in "Protocol to observe Janus droplet individual and multibody droplet swimming behavior".

Microscale Joule heater experiments. A microscale Joule heating device was fabricated using masked electron-beam evaporation (Kurt J. Lesker Lab-18) to deposit a three-layer architecture of $50 \mathrm{~nm} \mathrm{Ti,} 150$ $\mathrm{nm} \mathrm{Au}, 50 \mathrm{~nm} \mathrm{Ti}$ in the shape of two heating pads and protruding finger structures on top of a $1 \mathrm{~mm}$ thick glass slide (Figure S6). The microheater pads were $3 \times 6 \mathrm{~mm}$ rectangles and the fingers were 100 microns wide with a $20 \mathrm{~mm}$ distance from the pad to the tip of the finger and a spacing of $50 \mu \mathrm{m}$ between the fingers. To conduct droplet experiments, the device slide was taped to the bottom of a $90 \mathrm{~mm}$ diameter plastic Petri dish placed on top of the microscope stage and filled $15 \mathrm{~mL}$ of $0.5 \mathrm{wt} \%$ Triton surfactant solution with $0.1 \mathrm{M} \mathrm{NaCl}$ added as an electrolyte required for Joule heating. An AC current $(1 \mathrm{MHz}$, square wave, between $6 \mathrm{~V}$ and $20 \mathrm{~V}$ ) was applied to the microheater using a function generator (Koolertron CJDS66-15M) to create a thermal gradient radiating perpendicular from the heater fingers. Quantification of the thermal gradient was obtained using images from an infrared camera (FLIR SC6700) captured under the same experimental conditions used for droplet experiments on the microscope measured at a distance $515 \mu \mathrm{m}$ away from the heater pad along the finger. For speed measurements, 3050 iododecane-EFB Janus droplets were transferred using micropipette $10 \mathrm{~mm}$ away from the finger, roughly one third of the position up the finger length from the heater pad. Droplet speeds were tracked as a function of distance from the heater by analyzing video recording using custom Matlab code (same as mentioned in "Analysis of droplet external flows"). We note that while droplets approached the heater at different distances from the pad, only the distance from the fingers was tracked and the averaged values from five droplet measurements were used to assemble the data plotted in Figure $\mathbf{5 b}$. Convective flows generated from the heater device were revealed using $1 \mu \mathrm{m}$ latex tracer particles suspended in the surfactant solution. However, control experiments using 75-90 $\mu \mathrm{m}$ diameter polyethylene particles (1.02 $\mathrm{g} / \mathrm{cm}^{3}$ ) and single emulsion droplets composed of both iododecane and EFB oils ruled out convective flows or thermocapillary motion as primary cause for the Janus droplet motion so we presume that 
increased solubilization with increasing temperatures is the driver for the observed acceleration of Janus droplet speeds as they approached the heater.

Laser induced temperature gradient experiments. A continuous wave $780 \mathrm{~nm}$ laser (Coherent MIRA $900 \mathrm{~F}$ ) set to $250 \mathrm{~mW}$ (measured at back of objective, Nikon 40x $0.6 \mathrm{NA}$ ) was used to introduce thermal gradients into a dish containing surfactant solution via absorption of the beam by the aqueous liquid. Experiments were conducted in a glass bottom dish (glass $180 \mu \mathrm{m}$ in thickness) containing approximately $1.0 \mathrm{~mL}$ of $0.5 \mathrm{wt} \%$ Triton. Approximately 150 to 300 iododecane-EFB droplets were added to the dish using a micropipette. For droplet-gathering experiments using the laser beam, droplets were initially agitated to disperse them randomly throughout the dish. The beam was then turned on and focused near the dish center for the entirety of the experiment, generating a thermal profile emanating heat from the laser spot and drawing droplets towards the laser (Figure 5c, Video S4). For reorientation experiments using a scanned laser beam, a droplet cluster was initially formed by directly pipetting droplets into a central region of the imaging dish. Translation of the beam was directed using a combination of a nanoprecision XYZ stage (ASI RAMM/Modular infinity microscope system) and galvo-scanner oscillating mirrors (Nutfield Technology) resulting in moving laser focus that droplets continually reorient themselves towards (Figure 5d, Video S5). Imaging was accomplished using a CMOS camera (Point Grey Research, Blackfly) attached to the ASI stage. Further details of the optical setup are described in previous work ${ }^{7}$.

Analysis of droplet trail interactions. To study the dynamics of Janus droplets crossing the trails of neighboring droplets, approximately 100-200 iododecane-EFB droplets were pipetted into a glass-bottom dish containing $0.5 \mathrm{wt} \%$ Triton and then gently agitating to disperse droplets. This process randomized the droplet starting position and swimming orientations in order to ensure a lower density of trail overlap instances so that the effects of multiple overlapping trails was minimized. Janus droplets swimming in 0.5 $w t \%$ Triton were analyzed over nine videos capturing a viewing window size of $2230 \mu \mathrm{m}$ by $2230 \mu \mathrm{m}$ at a rate of 18 frames per second, for a total of 270 intersection events. Droplets were tracked and trajectories were reconstructed using a custom Matlab tracking code (same as for "Analysis of droplet external flows"). Intersections between trajectories were identified using existing algorithms. The magnitude decrease in swimming velocity when Janus droplets passed over trajectories left by previous droplets was then identified and correlated with the time lag between trajectory intersections. Each intersection event is characterized by particle $j$ crossing the trajectory of particle $i$ at position $\boldsymbol{x}_{i j}$ with a time lag of $\Delta t_{i j}=t_{j}-$ $t_{i}$. Where, $t_{i}$ and $t_{j}$ are the times when particles $i$ and $j$ occupied $\boldsymbol{x}_{i j}$, respectively. Times $t_{i}$ and $t_{j}$ were found by linear interpolation between the nearest video frames. To measure the speed decrease $V_{\min } / V_{0}$, each trajectory's positional data $\boldsymbol{x}_{j}$ was first smoothed using a Gaussian filter with a width of 20 frames $\left(\widehat{\boldsymbol{x}}_{j}\right)$. The instantaneous speed $V_{j, n}$ of particle $j$ at time $t_{n}$ was approximated as:

$$
V_{j, n}=\frac{\left|\widehat{x}_{j, n+1}-\widehat{x}_{j, n}\right|}{t_{n+1}-t_{n}}
$$

where $\widehat{\boldsymbol{x}}_{j, n}$ is the filtered position of particle $j$ at time $t_{n}$. $V_{\min }$ was estimated by finding the minimum value of $V_{j}$ on the interval $t_{j} \pm 5 \mathrm{~s}$. To estimate the average velocity $V_{0}$ of particle $j$ far from intersections, $t_{\max }$ was determined as the time of maximum $V_{j}$ on the interval $t_{j} \pm 5 \mathrm{~s} . V_{0}$ was estimated by performing a linear regression on the portion of positional data $\boldsymbol{x}_{j}$ contained in the interval $t_{\max } \pm 1 \mathrm{~s}$. To understand how the magnitude of speed decrease scales with the time lag between intersections, we fit all intersection events to an exponential recovery model (black line on Figure 6c):

$$
\frac{V_{\min }}{V_{0}}=A-B e^{-\frac{\Delta t}{\tau_{\text {trail }}},}
$$

with $A=0.905 \pm 0.031, B=0.266 \pm 0.037$, and $\tau_{\text {trail }}=38.0 \pm 14.1 \mathrm{~s}$ (95\% confidence). We also estimate the trail lifetime $\left(\tau_{\text {trail }}\right)$ by comparing the width of the droplet trail $2 \delta$ with the diffusivity of the micelles $D_{\text {micelle: }}$ :

$$
\tau_{\text {trail }} \sim \frac{\delta^{2}}{D_{\text {micelle }}}=\frac{\left(3 \times 10^{-5} \mathrm{~m}\right)^{2}}{4.9 \times 10^{-11} \mathrm{~m}^{2} \mathrm{~s}^{-1}} \sim 18 \mathrm{~s} .
$$

assuming that $2 \delta$ is equal to the diameter of the short-axis of the Janus droplet and $D_{\text {micelle }}$, for Triton is equal to $4.9 \times 10^{-11} \mathrm{~m}^{2} / \mathrm{s}$ at $20^{\circ} \mathrm{C}^{4}$. 


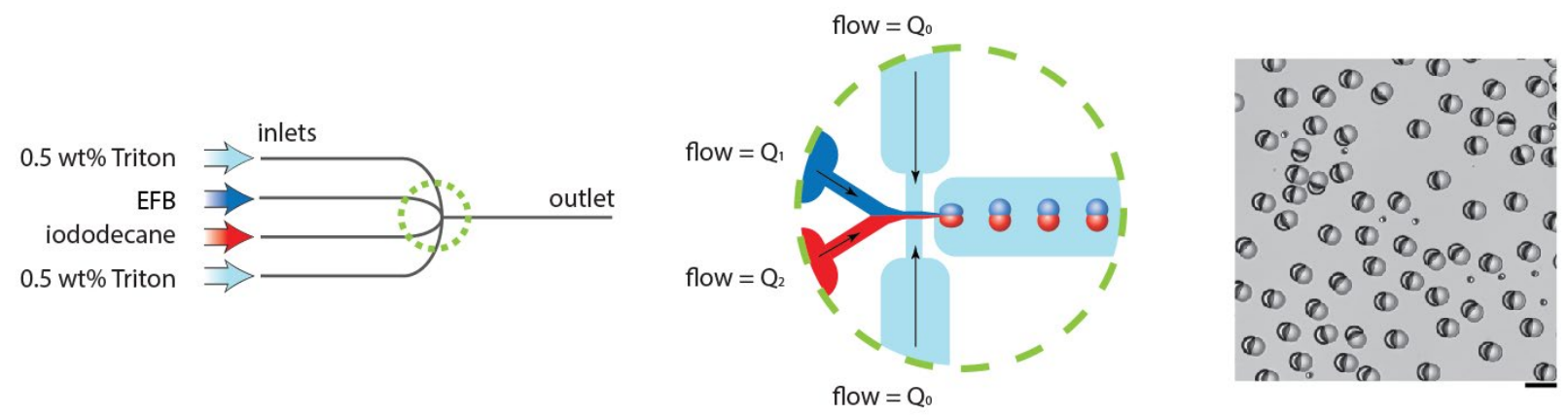

Figure S1. Microfluidic setup for producing biphasic oil-in-water Janus droplets. A glass microfluidic flow focusing chip is used having the general channel design as shown at left. The two oils are introduced in separate channels and droplets are pinched off at the junction as shown in the middle. Varying the flow rates of the four inlets allowed tuning of the droplet size and oil volume ratios. At left are images of iododecane-EFB droplets collected from the outlet of the microfluidic chip. Scale, $100 \mu \mathrm{m}$. 

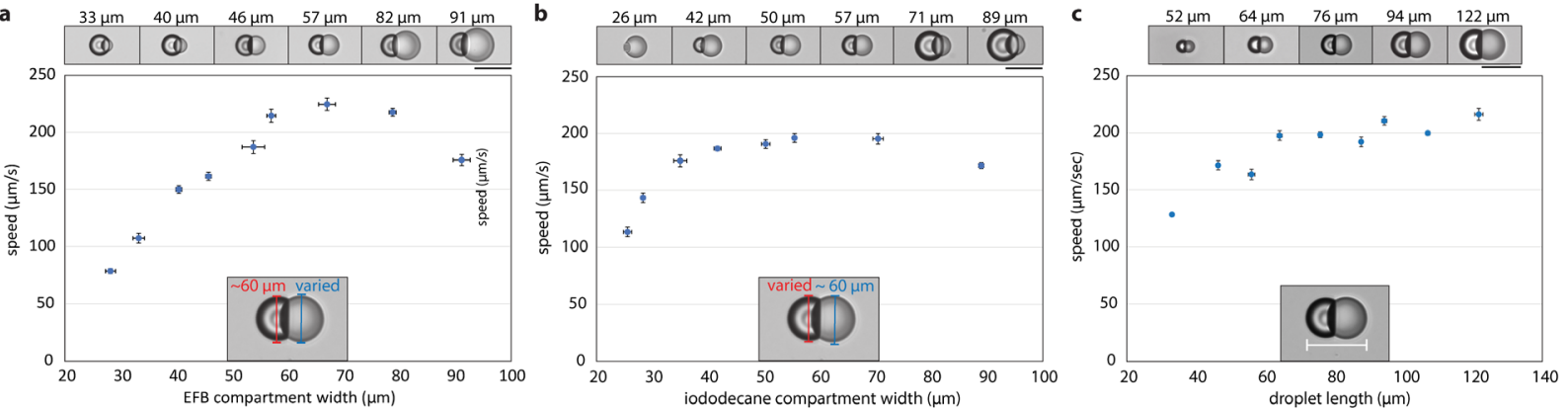

Figure S2. Janus droplet shape and size slightly affects swimming speeds. The speeds of iododecane-EFB Janus droplets in $0.5 \mathrm{wt} \%$ Triton vary with the diameter of the EFB compartment (a), the diameter of the iododecane compartment (b), and overall droplet size (c). Optical micrographs of Janus droplets show how the droplet shape changes. For all plots, each data point represents the average of five speed measurements obtained from the displacements of individual droplets over a 5 second period with standard deviation error bars. All droplets swim rightward, with the iododecane in the back and the EFB in the front. Scale, $100 \mu \mathrm{m}$. 

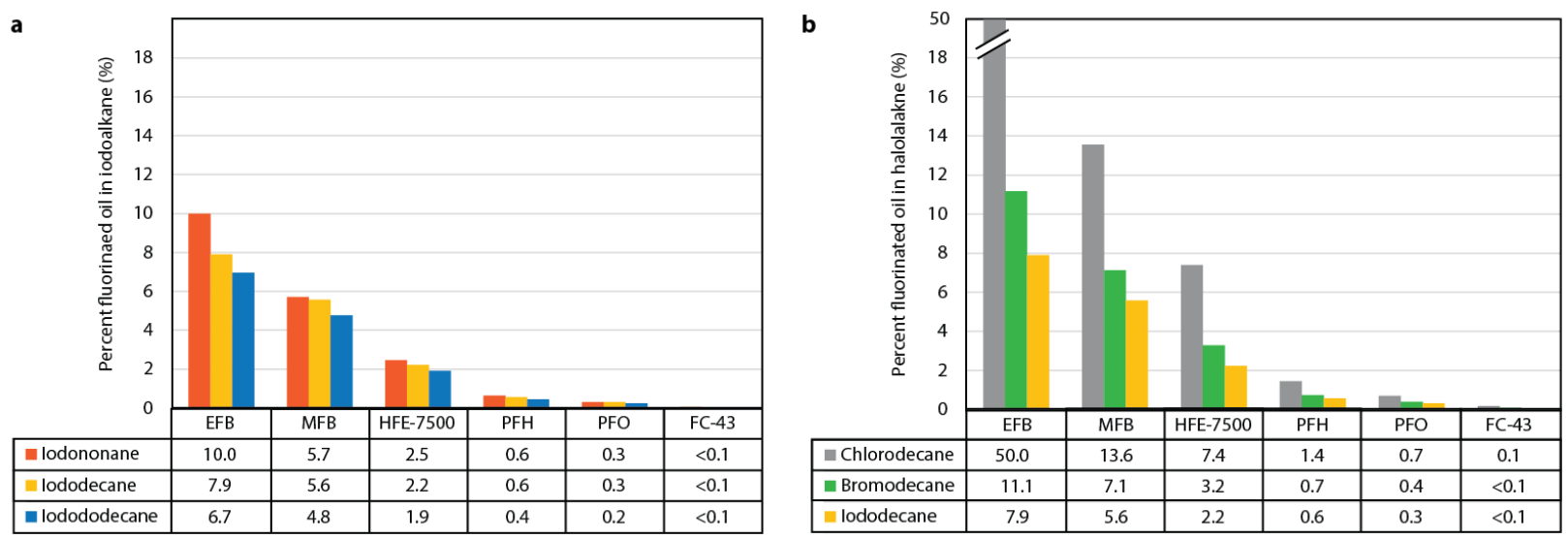

Figure S3. Degree of partitioning of the haloalkanes into the fluorinated oils. Degree of partitioning of each haloalkane into each fluorinated oil was determined using changes in refractive index as described in the Methods section "Oil partitioning measurement". The percentage is defined as the volume fraction of partitioned oil calculated based on the change in the refractive index of the haloalkane due to saturation by the fluorinated oil divided by the difference in refractive index between the two pure oils. More highly fluorinated oils had lower miscibility with the haloalkanes, as expected ${ }^{3}$. Please refer to Figure 2 for chemical structures of each of the fluorinated oils. Fully miscible oils are defined as having $50 \%$ partitioning. 
a

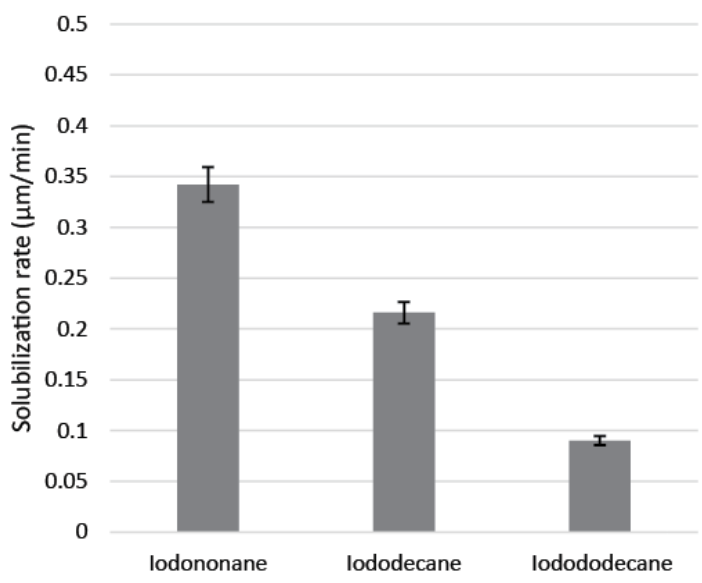

b

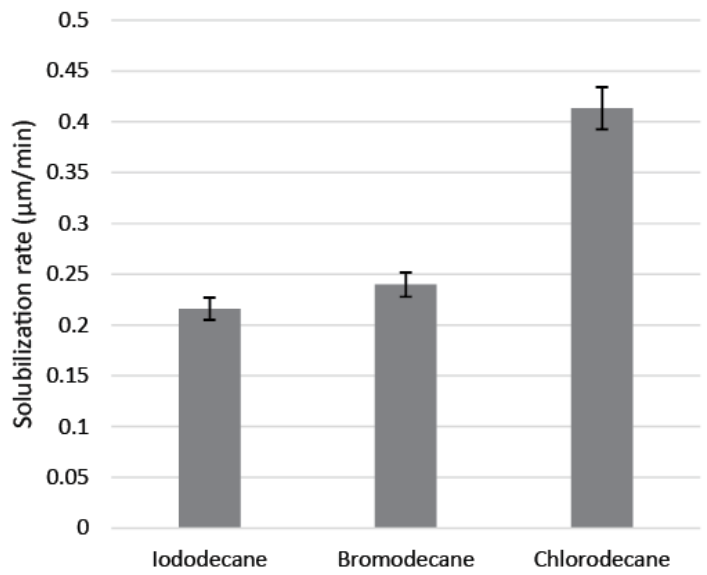

Figure S4. Solubilization rates of haloalkane droplets in $0.5 \mathrm{wt} \%$ Triton surfactant. Solubilization rates were obtained by observing the shrinkage of an isolated droplet of each oil over time. Solubilization rate is defined as the change in the droplet diameter per minute. Data represents the average and standard deviation for each oil as measured for three droplets. 

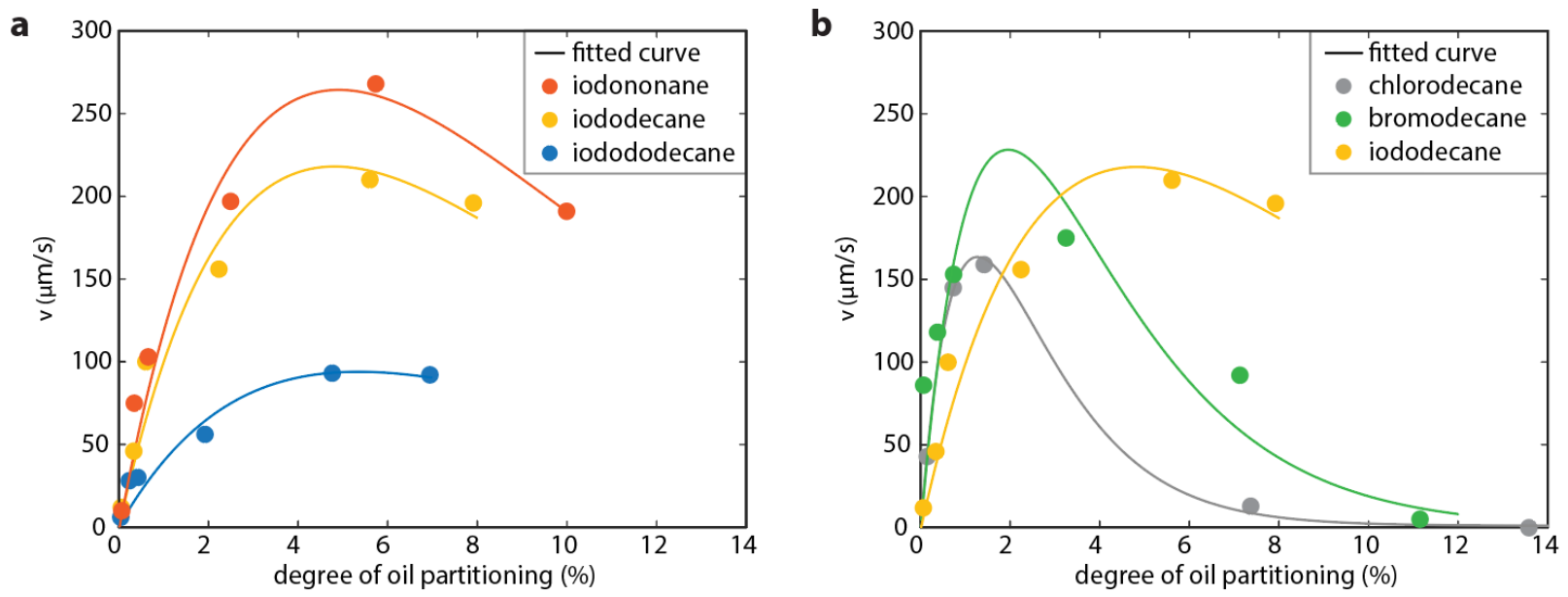

Figure S5. Trends in swimming speed vs. degree of partitioning for Janus droplets composed of haloalkane and fluorinated oils. For both (a) and (b), the speeds are the average speeds of Janus droplets in $0.5 \mathrm{wt} \%$ Triton from Figure 2 plotted as a function of the degree of partitioning for each oil pair taken from Figure S3. The data for each haloalkane with varying fluorinated oils is fitted empirically using a function with the form: $v=a * x * e^{-x / b}$, where $v$ is the swim speed, $x$ is the partitioning, and $a$ and $b$ are the fitting parameters which we propose are related to solubilization rate and the ability of the oil gradient to affect the interfacial tension, respectively. The optimal values for $a$ and $b$ determined by their minimized least square fits are: $a=146.7, b=4.90$ for iodononane, $a=123.2, b=4.81$ for iododecane, $a$ $=47.7, b=5.35$ for iodododecane, $a=318.5, b=1.95$ for bromodecane, and $a=348.3, b=1.27$ for chlorodecane. In (a), we observed that the peak swim speed $v_{\max }$ was higher for the shorter iodoalkanes which also is associated with faster oil solubilization rates (Figure S4). However, the partitioning $x$ associated with the peak swim speed remains relatively constant. In (b), we did not find a clear trend in peak swim speed $v_{\max }$ with solubilization rate but rather observed a leftward shift and narrowing of the fit curve when going from iodo- to bromo- to chloro-. Changes in the size and polarizability of the halogen atom apparently have notable impact on the oil molecular properties affecting factors such as surfactantoil interactions and the effectiveness of the oil-filled micelle gradients to modulate droplet interfacial tensions. 


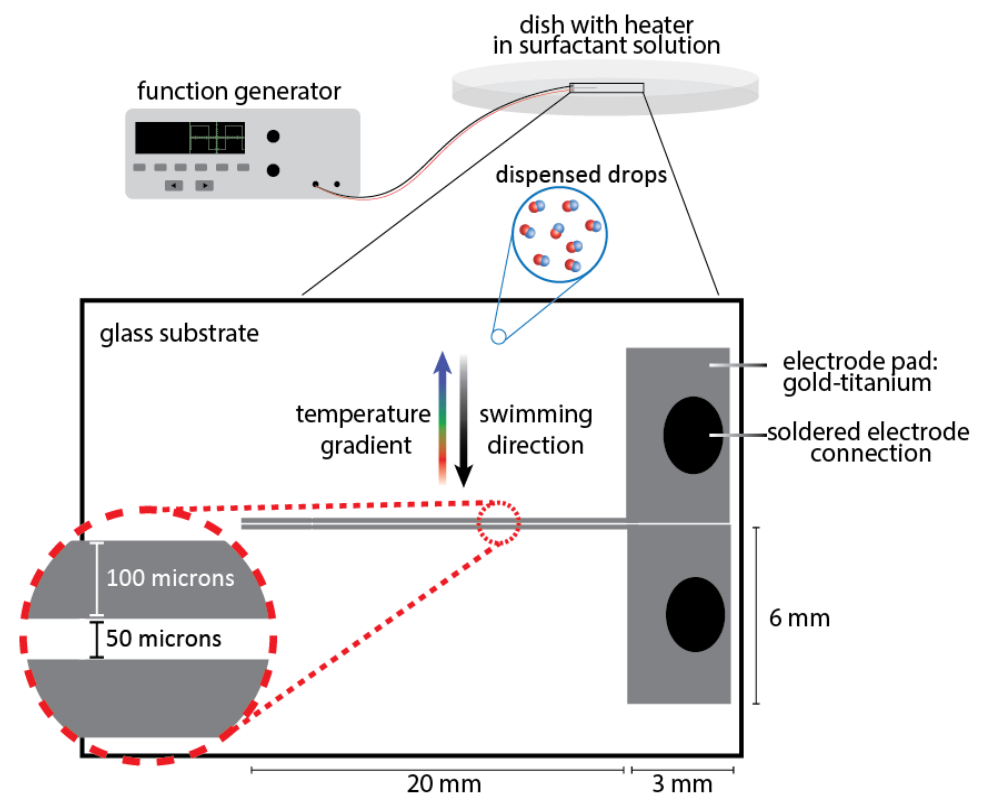

Figure S6. Schematic of microscale Joule heater and experimental setup. The microscale Joule heater consisted of gold-titanium electrodes on glass in the given dimensions. A function generator was used to apply a $1 \mathrm{MHz}$ square wave potential at $6-20 \mathrm{~V}$ to the electrodes while submerged in solution containing $0.5 \mathrm{wt} \%$ Triton and $0.1 \mathrm{M} \mathrm{NaCl}$ to generate heating around the electrodes. 
Table S1. Interfacial tensions between oils and aqueous surfactant, including both oil-saturated and unsaturated surfactant solution. The pendant drop method was used to measure interfacial tension. Measurements were taken every 3 seconds between 400 and 500 seconds after forming the drop to give an average and standard deviation. EFB is ethoxynonafluorobutane, MFB is methoxyperfluorobutane, and FC-43 is perfluorotributylamine.

\begin{tabular}{|l|l|l|l|}
\hline Oil & Surfactant & $\begin{array}{l}\text { Interfacial tension } \\
(\mathrm{mN} / \mathrm{m})\end{array}$ & Standard deviation \\
\hline $\begin{array}{l}\text { lododecane saturated } \\
\text { with EFB }\end{array}$ & $0.5 \mathrm{wt} \%$ Triton & 0.48 & 0.02 \\
\hline $\begin{array}{l}\text { EFB saturated with } \\
\text { iododecane }\end{array}$ & $0.5 \mathrm{wt} \%$ Triton & 1.80 & 0.05 \\
\hline $\begin{array}{l}\text { lododecane saturated } \\
\text { with EFB }\end{array}$ & $\begin{array}{l}\text { EFB and iododecane saturated } 0.5 \\
\text { wt\% Triton }\end{array}$ & 0.84 & 0.02 \\
\hline $\begin{array}{l}\text { EFB saturated with } \\
\text { iododecane }\end{array}$ & $\begin{array}{l}\text { EFB and iododecane saturated } 0.5 \\
\text { wt\% Triton }\end{array}$ & 2.00 & 0.1 \\
\hline $\begin{array}{l}\text { MFB saturated with } \\
\text { iododecane }\end{array}$ & 0.5 wt\% Triton & 3.56 & 0.02 \\
\hline $\begin{array}{l}\text { FC-43 saturated with } \\
\text { iododecane }\end{array}$ & $0.5 \mathrm{wt} \%$ Triton & 8.41 & 0.09 \\
\hline $\begin{array}{l}\text { MFB saturated with } \\
\text { iododecane }\end{array}$ & $\begin{array}{l}\text { lododecane saturated } 0.5 \mathrm{wt} \% \\
\text { Triton }\end{array}$ & 3.74 & 0.02 \\
\hline $\begin{array}{l}\text { FC-43 saturated with } \\
\text { iododecane }\end{array}$ & $\begin{array}{l}\text { lododecane saturated } 0.5 \mathrm{wt} \% \\
\text { Triton }\end{array}$ & 8.37 & 0.12 \\
\hline
\end{tabular}


Video S1 (separate file). Janus droplets composed of iododecane and EFB swim in 0.5 wt\% Triton X100 surfactant solution. Speed, $5 x$. Scale, $250 \mu \mathrm{m}$.

Video S2 (separate file). Droplets composed of dibromooctane and fluorosilicone oil in $0.5 \mathrm{wt} \%$ Triton X100 reverse swimming orientation over time. Speed, 10x. Scale, $250 \mu \mathrm{m}$.

Video S3 (separate file). Marangoni-driven flow transition from the forward to reverse swimming direction outside a dibromooctane-fluorosilicone Janus droplet visualized by tracer particle motion in $0.3 \mathrm{wt} \%$ Triton. Speed, 5x. Scale, $100 \mu \mathrm{m}$.

Video S4 (separate file). A focused $780 \mathrm{~nm}$ laser, position marked by the red dot, generates local heating. lododecane-EFB droplets in $0.5 \mathrm{wt} \%$ Triton gather near the laser focal point. Speed, 10x. Scale, $100 \mu \mathrm{m}$.

Video S5 (separate file). A focused $780 \mathrm{~nm}$ laser is scanned up and down across the field of view and translated laterally. As the laser is scanned over the iododecane-EFB droplets in $0.5 \mathrm{wt} \%$ Triton, the droplets reorient in response. Speed, 3x. Scale, $100 \mu \mathrm{m}$.

Video S6 (separate file). lododecane-EFB droplets in $0.5 \mathrm{wt} \%$ Triton leave behind an invisible trail of solubilized oil in micelles that affect the motion of other droplets that cross the trail. Trails of the droplets are drawn in the video with the color change indicating the lifetime of the trail. Recent trails are colored in yellow while older trails are purple. Droplet trail markings disappear after 60 seconds. Speed, 5x. Scale, $250 \mu \mathrm{m}$.

Video S7 (separate file). Janus droplets of iododecane-methoxyperfluorobutane in $0.5 \mathrm{wt} \%$ Triton spontaneously form coordinated clusters that spin directionally based on the cluster symmetry. Speed, 2x. Scale, $250 \mu \mathrm{m}$.

Video S8 (separate file). Tracer particles were used to visualize the flows around spinning iododecanemethoxyperfluorobutane droplet clusters in $0.5 \mathrm{wt} \%$ Triton. Left video is focusing in the plane of the droplets and the right video is focusing above the plane of the droplets. Speed, 1.5x. Scale, $50 \mu \mathrm{m}$.

Video S9 (separate file). An iododecane-EFB droplet wetted to the glass substrate is unable to swim and instead pumps the surrounding fluid which is visualized by the motion of tracer particles. The flow direction proceeds from the EFB compartment towards the iododecane compartment. Speed. 5x. Scale, $50 \mu \mathrm{m}$. 


\section{SI References}

1 Meredith, C. H. et al. Predator-prey interactions between droplets driven by non-reciprocal oil exchange. Nature Chemistry 12, 1136-1142 (2020).

2 Carroll, B. J. The kinetics of solubilization of nonpolar oils by nonionic surfactant solutions. Journal of Colloid and Interface Science 79, 126-135 (1981).

3 Gladysz, J. A., Curran, D. P. \& Horváth, I. T. Handbook of fluorous chemistry. (John Wiley \& Sons, 2006).

4 Paradies, H. H. Shape and size of a nonionic surfactant micelle. Triton X-100 in aqueous solution. The Journal of Physical Chemistry 84, 599-607 (1980).

5 Crocker, J. C. \& Grier, D. G. Methods of digital video microscopy for colloidal studies. Journal of Colloid and Interface Science 179, 298-310 (1996).

6 Thielicke, W. \& Stamhuis, E. J. PIVlab-time-resolved digital particle image velocimetry tool for MATLAB. Published under the BSD license, programmed with MATLAB 7, R14 (2014).

$7 \quad$ Kindle, C. et al. Direct laser writing from aqueous precursors for nano to microscale topographical control, integration, and synthesis of nanocrystalline mixed metal oxides. ACS Applied Nano Materials 2, 2581-2586 (2019). 